\title{
MEASURES ON TOPOLOGICAL SPACES
}

\section{by}

\section{Susumu OKADA}

A thesis submitted for the degree of Master of Science at the Australian National University.

Canberra, July 1979. 


\section{STATEMENT}

Except where otherwise stated, the material contained in this thesis is the product of my own research.

Susumu Okada

Susumu OKADA 


\section{ACKNOWLEDGEMENTS}

My warmest thanks go to my supervisor, Dr S. Yamamuro for his many suggestions to my work and constant encouragement.

For awarding me a research scholarship and providing an excellent environment I thank the Australian National University.

I am indebted to Profesosr I. Amemiya for suggesting an improvement of \$3.2, to Dr J. Dawson for helping me with my text, to Professor W. Moran for showing a helpful example, Example 5.3.9, and to Professor Y. Yamasahi for useful advice with some results in $\$ 2.3$ and $\$ 4.1$.

Many thanks are due to Mr Y. Okazaki, who informed me of many results and who provided me with much encouragement.

Finally I would like to express my gratitude to Mrs Barbara Geary for her excellent typing under trying circumstances, and to Miss Sybil Stuckey for her kindness and help during the past two years. 


\section{ABSTRACT}

This thesis examines $\tau$-smooth measures and supports of Borel measures. A Radon measure is a $\tau$-smooth Borel measure and has a support. These two characteristics, $\tau$-smoothness and the existence of a support, can not be set aside when we investigate interaction between topological spaces and measures on them. In the field of Baire measure theory, one of the main topics has been the study of these characteristics.

In order to study $\tau$-smooth measures in a general setting, we present the concept of pre-Radon measures, which are locally bounded, regular, $\tau$-smooth Borel measures. Further, some pre-Radon extension theorems are given, which are fundamental tools for developing pre-Radon measure theory. The method used to prove these theorems is also used to prove Kolmogorov's extension theorem for infinite Radon measures. The pre-Radon extension theorems are applied to prove Fubini's theorem for $\sigma$-finite pre-Radon measures, which is needed in order to prove that the infinite product of pre-Radon probability measures can be extended to a pre-Radon probability measure. As another application of the pre-Radon extension theorems, we show that some cylindrical measures on locally convex spaces can be extended to Radon or pre-Radon measures.

When we consider families of measures on topological spaces, the existence of supports implies certain $\tau$-smoothness conditions. Here, the countable union and the hereditariness of spaces which are, for example, measure-compact or Borel measure-compact, are investigated.

On some topological spaces such as paracompact spaces, every finite Borel measure has a Lindelöf support. We say that a topological space having this property is an SL-space. Metacompact spaces and $\theta$-paracompact spaces are SL-spaces, but $\theta$-refinable spaces are not always SL-spaces. 


\section{TABLE OF CONTENTS}

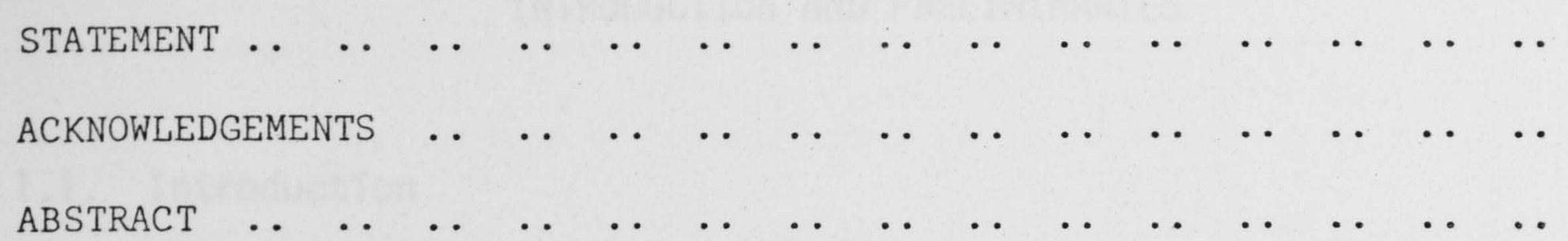

CHAPTER I: INTRODUCTION AND PRELIMINARIES $\ldots$

1.1 Introduction $\ldots$

1.2 Preliminaries and fundamental results $\ldots \quad \ldots \quad \ldots \quad 3$

$\begin{array}{llllllllllllllll}1.3 & \sigma \text {-algebras } \ldots & \ldots & \ldots & \ldots & \ldots & \ldots & \ldots & \ldots & \ldots & \ldots & & 13\end{array}$

CHAPTER II: EXTENSION THEOREMS

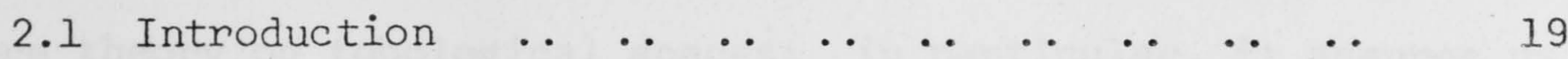

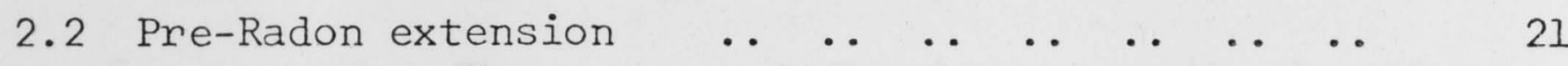

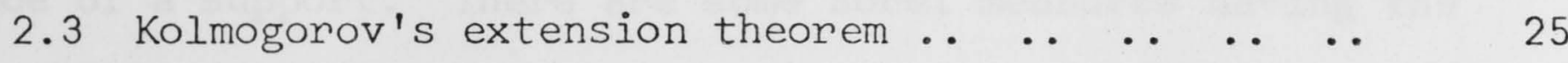

CHAPTER III: APPLICATIONS OF PRE-RADON EXTENSION THEOREMS $\quad \ldots \quad \ldots \quad 29$

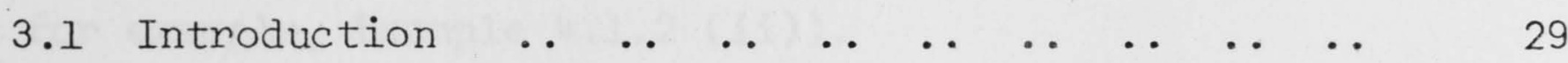

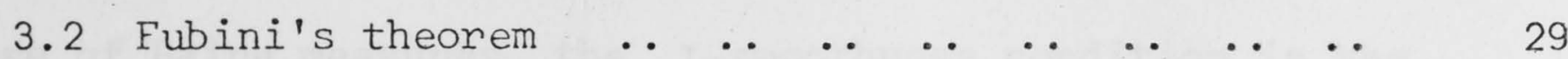

3.3 Infinite product of pre-Radon measures .. $\ldots . .34$

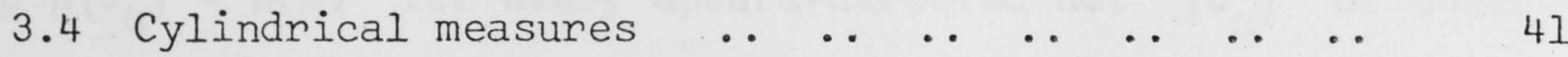

CHAPTER IV: MEASURE-COMPACTNESS AND BOREL MEASURE-COMPACTNESS . . 46

4.1 Introduction and preliminary results $\ldots \quad \ldots \quad \ldots \quad 46$

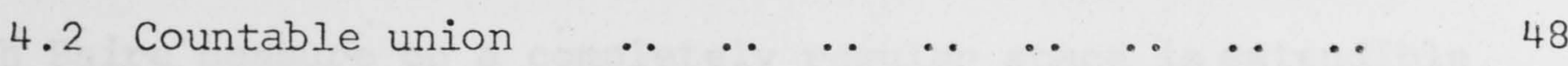

4.3 Hereditariness ..

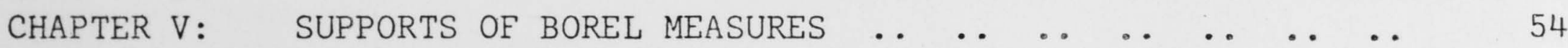

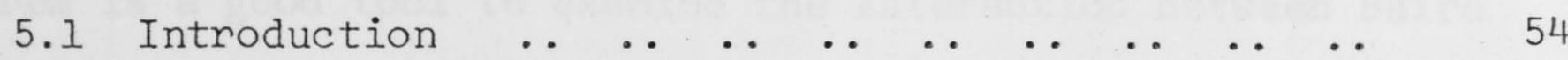

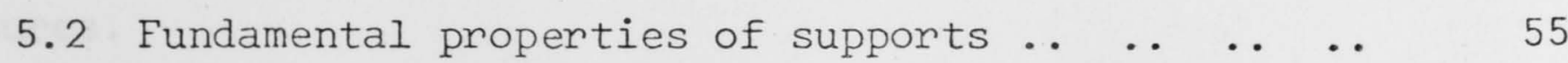

$\begin{array}{lllllllllllll}5.3 & \text { Lindelöf supports } & \ldots & \ldots & \ldots & \ldots & \ldots & \ldots & \ldots & \ldots & & 59\end{array}$

$\begin{array}{lllllllllllll}5.4 & \text { SL-Spaces } & \ldots & \ldots & \ldots & \ldots & \ldots & \ldots & \ldots & \ldots & \ldots & \ldots & 63\end{array}$

$\begin{array}{llllllllllllllllll}\text { REFERENCES } & \ldots & \ldots & \ldots & \ldots & \ldots & \ldots & \ldots & \ldots & \ldots & \ldots & \ldots & \ldots & \ldots & \ldots & & 66\end{array}$ 


\section{CHAPTER 1}

\section{INTRODUCTION AND PRELIMINARIES}

\subsection{Introduction}

One of the characteristics of Radon measures on arbitrary topological spaces developed by Bourbaki [5] and Schwartz [42] is the following $\tau$-smoothness condition: $\sup _{\alpha} \mu\left(G_{\alpha}\right)=\mu\left(U_{\alpha} G_{\alpha}\right)$ for every upward-directed net $\left\{G_{\alpha}\right\}$ of open subsets. This condition is indispensable in many cases when we study measure theory on topological spaces; in particular, it assures us of the existence of a support. There are some Borel measures having the $\tau$-smoothness condition: $\tau$-smooth Borel measures, which are not Radon measures (see, for example, Example 4.1.2 (ii)).

In the case of Baire measures, the $\tau$-smoothness condition is the following: $\sup _{\alpha} \mu\left(U_{\alpha}\right)=\mu(X)$ for every upward-directed net $\left\{U_{\alpha}\right\}$ of cozero sets such that $U_{\alpha} U_{\alpha}=X$. It is shown by Knowles [23] and Kirk [22] that every $\tau$-smooth Baire measure on a completely regular space is extendible to a unique $\tau$-smooth Borel measure (Theorem 2.1.2). This Knowles-Kirk's extension theorem is a good tool to examine the interaction between Baire and Borel measures.

This thesis is devoted to the study of some aspects of $\tau$-smooth measures, and supports of Borel measures. Both are closely related ( $c f$. Gardner [10], Moran [30], [31] and Varadarajan [47]).

We introduce the notion of pre-Radon measures, which are regular, locally bounded $\tau$-smooth Borel measures on topological spaces. Infinite $\tau$-smooth Borel measures are not always regular, while every finite $\tau$-smooth Borel measure on a regular space is regular (Gardner [10, Theorem 5.4]). Consequently every Radon measure is not necessarily a pre-Radon measure 
although every $\sigma$-finite Radon measure is a pre-Radon measure. In fact, there are some Radon measures which are not outer regular. However, the outer regularity is essential to develop pre-Radon measure theory. Fremlin [9] has given the concept of quasi-Radon measures which have $\tau$-smoothness, and dispenses with the outer regularity. But he uses some extra conditions. As for the relationship between pre-Radon measures and quasi-Radon measures, see Theorem 1.2.10.

On a perfectly normal space, the Baire field is identical to the Borel field. In particular, this is valid on a metric space. However, it does not hold, in general; Proposition 1.3.1, Corollary 1.3.5 and Corollary 1.3.8 give examples that the Baire fields are different from the Borel fields. In the latter half of $\$ 1.3$, we present some results on the relationships between cylindrical o-algebras, Baire fields and Borel fields on locally convex spaces.

In Chapter II we prove pre-Radon extension theorems, and Kolmogorov's extension theorem for infinite Radon measures. We treat the pre-Radon extension in a general setting, so that Knowles-Kirk's $\tau$-smooth extension theorem can be a corollary. Kolmogorov's extension theorem for infinite Radon measures is proved by using the same technique as in pre-Radon extension theorems.

In Chapter III we apply the pre-Radon extension theorems to some cases. First we prove Fubini's theorem. This is used to prove that the infinite product of pre-Radon probability measures can be extended to a unique preRadon probability measure. Furthermore, some cylindrical measures are extended to pre-Radon or Radon measures by applying the pre-Radon extension theorems.

In Chapter IV, we study some properties of measure-compact, strongly measure-compact, Borel measure-compact, $B^{*_{-}}, H^{*_{-}}$and Radon spaces: we investigate whether they are closed by the operation of countable union; and 
whether their subspaces inherit the same properties from them.

In Chapter $\mathrm{V}$, we introduce a new class of topological spaces on which every Borel measure has a Lindelöf support. They are called SL-spaces. In the case of Baire measures, Varadarajan [47, Theorem I.27] has proved that every Baire measure on a paracompact space has a Lindelöf support; more generally, Moran [32, Theorem 5.1] has proved that every Baire measure on a metacompact space has a Lindelöf support. It is shown that every metacompact space is an $S L$-space, and that every $\theta$-paracompact space is also an $S L$-space (for the definition of $\theta$-paracompactness, see $\$ 5.3$ ). However, all $\theta$-refinable spaces are not $S L$-spaces.

\subsection{Preliminaries and Fundamental Results}

As usual, let us reserve the symbols $\mathbb{N}$ for the set of natural

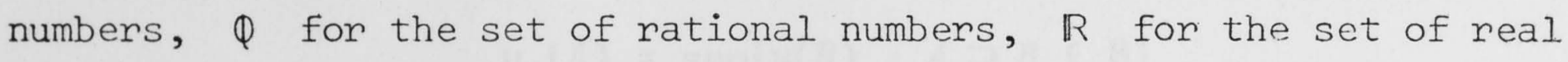
numbers and $\mathbb{R}^{+}$the set of non-negative real numbers. When we regard $\mathbb{R}$ as a topological space, the topology is the euclidean topology.

Let $X$ be a set and $A$ a subset of $X$. Then $X_{A}$ denotes the characteristic function of $A$, that is,

$$
X_{A}(x)=\left\{\begin{array}{lll}
1 & \text { if } & x \in A, \\
0 & \text { if } & x \notin A .
\end{array}\right.
$$

Let $\left\{A_{\alpha}\right\}$ be a family of subsets of $X$. Then $A_{\alpha} \downarrow A$ means that

(i) $\left\{A_{\alpha}\right\}$ is a downward-directed net, that is, $A_{\alpha} \subset A_{\beta}$ for

$$
\alpha \geq \beta \text {, and }
$$

(ii) $A=\underset{\alpha}{\bigcap_{\alpha}} A_{\alpha}$.

Similarly we can define $A_{\alpha} \uparrow A$.

Let $X$ be a set and $B$ a $\sigma$-algebra of subsets of $X$. Then we call the pair $(X, B)$ a measurable space. Let $\mu$ be a non-negative, extended 
real-valued, $\sigma$-additive set function on $B$. Then we call $\mu$ a measure, and the triple $(X, B, \mu)$ a measure space. We say that a measure $\mu$ is

(i) finite if $\mu(X)<\infty$;

(ii) $\sigma$-finite if there exists a sequence $\left\{A_{n}\right\}_{n \in \mathbb{N}} \subset B$ such that $X=\underset{n \in \mathbb{N}}{U} A_{n}$ and $\mu\left(A_{n}\right)<\infty$ for all $n \in \mathbb{N}$;

(iii) semi-finite if $\mu(A)=\sup \{\mu(B): A \supset B \in B, \mu(B)<\infty\}$ for all $A \in B$.

Let $m$ be an extended real-valued set function on a family $A$ of subsets of a set. Given a subfamily $B$ of $A,\left.m\right|_{B}$ denotes the restriction of $m$ to $B$.

Given a measure space $(X, B, \mu)$ let

$$
\mu^{*}(A)=\inf \{\mu(B): A \subset B \in B\},
$$

and let

$$
\mu_{*}(A)=\sup \{\mu(B): A \supset B \in B\} .
$$

We say that the set function $\mu^{*}$ (respectively $\mu_{*}$ ) on the family $2^{X}$ of all subsets of $X$ is the outer measure (respectively inner measure) derived from $\mu$. For a subset $A$ of $X,\{A \cap B: B \in B\}$ is a $\sigma$-algebra on $A$, which we denote by $A \cap B$. In particular, if $A \in B$, we can define the restriction $\mu_{A}$ of $\mu$ to $A$ by $\mu_{A}(A \cap B)=\mu(A \cap B)$ for each $B \in B$. We sometimes write $\left.\mu\right|_{A}$ instead of $\mu_{A}$ to avoid confusion. A subset $Y$ of $X$ is said to be $\mu$-thick if $\mu_{*}\left(Y^{c}\right)=0$. Then $\left.\mu^{*}\right|_{Y \cap B}$ is a measure on $(Y, Y \cap B)$, which we call the restriction of $\mu$ to $Y$ and denote by $\mu_{Y}$ or $\left.\mu\right|_{Y}$. Consider a subset $A$ of $X$ such that $0<\mu^{*}(A)<\infty$. Then there exists a $B \in B$ such that $A \subset B$ and $\mu(B)=\mu^{*}(A)$. Since $\mu_{*}(B-A)=0, A$ is $\mu_{B}$-thick in $B$. Hence we can define the restriction $\left(\mu_{B}\right)_{A}$ of $\mu_{B}$ to $A$. Then we have 
$\left(\mu_{B}\right)_{A}(E)=\mu^{*}(E)$ for all $E \in A \cap B$, and $\left(\mu_{B}\right)_{A}$ is independent of the choice of $B$. This $\left(\mu_{B}\right)_{A}$ is also called the restriction of $\mu$ to $A$, and denoted by $\mu_{A}$ or $\left.\mu\right|_{A}$.

Let $\left\{\left(X_{\lambda}, B_{\lambda}, \mu_{\lambda}\right)\right\}_{\lambda \in \Lambda}$ be a family of measure spaces such that $\mu_{\lambda}\left(X_{\lambda}\right)=1$; that is, every $\mu_{\lambda}$ is a probability measure. Then $\underset{\lambda \in \Lambda}{\otimes} B_{\lambda}$ denotes the product $\sigma$-algebra on $\prod_{\lambda \in \Lambda} x_{\lambda} ;$ namely, the minimal $\sigma$-algebra of subsets of $\prod_{\lambda \in \Lambda} x_{\lambda}$ which makes each projection $p_{\lambda}$ of $x$ to $x_{\lambda}$ measurable. There exists a unique probability measure $\underset{\lambda \in \Lambda}{\otimes} \mu_{\lambda}$ on $\left(\prod_{\lambda \in \Lambda} x_{\lambda}, \underset{\lambda \in \Lambda}{\otimes} B_{\lambda}\right)$ such that

$$
\left(\underset{\lambda \in \Lambda}{\otimes} \mu_{\lambda}\right)(A)=\prod_{i=1}^{n} \mu_{\lambda_{i}}\left(A_{\lambda_{i}}\right)
$$

for each set $A=\prod_{i=1}^{n} A_{\lambda_{i}} \times \prod_{\lambda \neq \lambda_{i}} x_{\lambda} \in \underset{\lambda \in \Lambda}{\otimes} B_{\lambda}$ (see, for example, Halmos [16, Theorem 38.B]). This measure $\underset{\lambda \in \Lambda}{\otimes} \mu_{\lambda}$ is called the product measure.

Let $(X, A)$ and $(Y, B)$ be two measure spaces and $f: X \rightarrow Y$ a measurable map. For a measure $\mu$ on $(X, A)$, we define the image measure $f(\mu)$ of $\mu$ by $f$ as follows:

$$
f(\mu)(B)=\mu\left(f^{-1}(B)\right)
$$

for all $B \in B$.

Let $X$ be a set. A family $U$ of subsets of $X$ is called a paving if it satisfies the following conditions:

$$
\begin{aligned}
& \text { (i) } \emptyset \in U \text {; } \\
& \text { (ii) } \underset{U \in U}{U} U=X \text {; }
\end{aligned}
$$


(iii) if $U_{1}, U_{2} \in U$, then $U_{1} \cap U_{2} \in U$ and $U_{1} \cup U_{2} \in U$.

Let us denote by $R[U]$ (respectively $A[U]$ ) the ring (respectively

algebra) generated by $U$. The following lemma is well-known.

LEMMA 1.2.1. Let $A$ be a subset of $X$. Then $A \in R[U]$ if and only if there exist $V_{i}, W_{i} \in U(i=1,2, \ldots, n)$ such that

$$
\begin{aligned}
& \text { (i) } V_{i} \subset W_{i} \text { for alz } i=1,2, \ldots, n ; \\
& \text { (ii) }\left(W_{i}-V_{i}\right) \cap\left(W_{j}-V_{j}\right)=\varnothing \text { if } i \neq j ; \\
& \text { (iii) } A=\bigcup_{i=1}^{n}\left(W_{i}-V_{i}\right) .
\end{aligned}
$$

Suppose that $X$ is a set and $A$ is a family of subsets of $X$. Then $\sigma(A)$ denotes the $\sigma$-algebra generated by $A$.

Let $X$ be a topological space. We reserve the symbols $O(X)$ for the family of open subsets of $X, F(X)$ for the family of closed subsets of $X$, and $K(X)$ for the family of compact subsets of $X$. A $G_{\delta}$-subset is a countable intersection of open subsets; an $F_{\delta}$-subset is a countable union of closed subsets. Let $C(X)$ denote the ring of all real-valued continuous functions on $X$, and let

$$
Z(X)=\left\{f^{-1}(0): f \in C(X)\right\}
$$

and

$$
U(X)=\left\{f^{-1}(0)^{c}: f \in C(X)\right\} .
$$

We call the elements of $Z(X)$ (respectively $U(X)$ ) zero sets (respectively cozero sets). Note that

$$
Z(X)=\left\{f^{-1}(F): f \in C(X), F \in F(\mathbb{R})\right\}
$$

and

$$
U(X)=\left\{f^{-1}(U): f \in C(X), U \in O(\mathbb{R})\right\} .
$$

For $x \in X$, let $V(x)$ denote the family of all open neighbourhoods of $x$ in $X$. For $A \subset X$ and $x \in A, V_{A}(x)$ stands for the family of all open 
neighbourhoods of $x$ in $A$.

From now on, all topological spaces are supposed to be Hausdorff spaces.

Let $X$ be a topological space. Let $B^{*}(X)=\sigma(U(X))$ and $B(X)=\sigma(O(X))$. We say that $B^{*}(X)$ is the Baire field of $X$ and $B(X)$ the Borel field of $X$. It is obvious that $B^{*}(X)=\sigma(Z(X))$ and $B(X)=\sigma(F(X))$. In general, $B^{*}(X)$ is a sub- $\sigma$-algebra of $B(X)$.

In each subset $A$ of a topological space $X, B(A)=A \cap B(X)$; however $B^{*}(A)=A \cap B^{*}(X)$ does not always hold. We say that $A$ is Baireembedded if $B^{*}(A)=A \cap B^{*}(X)$. Recall that $A$ is called $C^{*}$-embedded if, for each real-valued, bounded continuous function $f$ on $A$, there is a $g \in C(X)$ such that $\left.g\right|_{A}=f$ on $A$. If $A$ is $C^{*}$-embedded, then $A$ is Baire-embedded. In particular, each closed subset of a normal space is Baire-embedded.

Let $X$ be a topological space. A finite measure on $\left(X, B^{*}(X)\right)$ is said to be a Baire measure. Every Baire measure $\mu$ satisfies the following regular condition:

$$
\begin{aligned}
\mu(A) & =\sup \{\mu(Z): A \supset Z \in Z(X)\} \\
& =\inf \{\mu(U): A \subset U \in U(X)\}
\end{aligned}
$$

for all $A \in B^{*}(X)$.

DEFINITION 1.2.2. Let $\mu$ be a Baire measure on a topological space $X$. We say that $\mu$ is

(i) $\tau$-smooth if $\mu\left(Z_{\alpha}\right) \downarrow 0$ for every net $\left\{z_{\alpha}\right\} \subset Z(X)$ such that $z_{\alpha} \downarrow \varnothing ;$

(ii) tight if $\mu(X)=\sup \left\{\mu^{*}(K): K \in K(X)\right\}$.

It is evident that all tight measures are $\tau$-smooth.

The support of a Baire measure is defined by

$$
S^{*}(\mu)=\{x \in X: \mu(V)>0 \text { for every } V \in V(x) \cap U(X)\} \text {. }
$$


Note that $S^{*}(\mu)=\cap\{Z \in Z(X): \mu(Z)=\mu(X)\}$. It follows from the definitions that a non-zero $\tau$-smooth Baire measure has a non-empty support. An extended real-valued measure on the Borel field $B(X)$ is called a Borel measure.

DEFINITION 1.2.3. Let $\mu$ be a Borel measure on a topological space $X$. We say that $\mu$ is

(i) inner regular if $\mu(A)=\sup \{\mu(F): A \supset F \in F(X)\}$ for each $A \in B(X)$ such that $\mu(A)<\infty$;

(ii) outer regular if $\mu(A)=\inf \{\mu(U): A \subset U \in O(X)\}$ for each $A \in B(X)$;

(iii) compact inner regular if $\mu(A)=\inf \{\mu(K): A \supset K \in K(X)\}$ for each $A \in B(X)$ such that $\mu(A)<\infty$;

(iv) regular if $\mu$ is both inner and outer regular.

Now consider the following condition:

(i)' $\mu(U)=\sup \{\mu(F): U \supset F \in F(X)\}$ for each $U \in O(X)$ such that $\mu(U)<\infty$.

Then (i)' and (ii) imply (i) by the same way as in the proof of Theorem (11.32) of Hewitt and Ross [18]; that is, (i) and (ii) are equivalent to (i)' and (ii).

Note that even if a Borel measure $\mu$ on $X$ is regular, $\mu$ does not necessarily satisfy the following condition:

(i)" $\mu(A)=\sup \{\mu(F): A \supset F \in F(X)\}$ for each $A \in B(X)$.

If $\mu$ is finite, then its inner regularity and outer regularity are equivalent; otherwise this does not necessarily hold (see Gruenhage and Pfeffer [13, Example 6, Example 7]). The following theorem gives a sufficient condition for an inner regular Borel measure to be outer regular.

THEOREM 1.2.4. Let $\mu$ be a Borel measure on a topological space $X$ such that $X=U_{n \in \mathbb{N}} U_{n}$ for some sequence $\left\{U_{n}\right\}_{n \in \mathbb{N}} \subset O(X)$ with $\mu\left(U_{n}\right)<\infty$. 
If $\mu$ is inner regular, then $\mu$ is outer regular.

Proof. Given $A \in B(X)$ and $\varepsilon>0$, there is an $F_{n} \in F(X)$ such that $F_{n} \subset A^{c} \cap U_{n}$ and $\mu\left(A^{c} \cap U_{n}^{-F} n\right)<\varepsilon / 2^{n}$ for all $n \in \mathbb{N}$. Let $V_{n}=U_{n} \cap F_{n}^{c} \in O(X)$. Then it follows that

$$
\mu\left(\bigcup_{n \in \mathbb{N}} V_{n}-A\right) \leq \sum_{n \in \mathbb{N}} \mu\left(V_{n}-U_{n} \cap A\right)<\varepsilon .
$$

Hence $\mu$ is outer regular. //

COROLLARY 1.2.5. Let $\mu$ be an inner regular Borel measure on a o-compact, locally compact space $X$ such that $\mu(K)<\infty$ for all $K \in K(X)$. Then $\mu$ is outer regular.

There is a sufficient condition for an outer regular Borel measure to be inner regular.

THEOREM 1.2.6. Let $\mu$ be a $\sigma$-finite, outer regular Borel measure on a topological space $X$. Then $\mu$ is inner regular.

Proof. There is a sequence $\left\{E_{n}\right\}_{n \in \mathbb{N}} \subset B(X)$ such that $X=\underset{n \in \mathbb{N}}{U} E_{n}$ and $\mu\left(E_{n}\right)<\infty$ for all $n \in \mathbb{N}$. For each $A \in B(X)$, each $\varepsilon>0$ and each $n \in \mathbb{N}$, there is a $U_{n} \in O(X)$ such that $U_{n} \supset A^{c} \cap E_{n}$ and

$$
\begin{aligned}
& \mu\left(U_{n}-A^{c} \cap E_{n}\right)<\varepsilon / 2^{n} \text {. Then } \\
& \mu\left(A-n_{n \in \mathbb{N}} U_{n}^{c}\right)=\mu\left(U_{n \in \mathbb{N}}^{U} U_{n}-\bigcup_{n \in \mathbb{N}}\left(A^{c} \cap E_{n}\right)\right) \leq \sum_{n \in \mathbb{N}} \mu\left(U_{n}-A^{c} \cap E_{n}\right)<\varepsilon .
\end{aligned}
$$

Thus $\mu$ is inner regular. //

DEFINITION 1.2.7. Let $\mu$ be a Borel measure on a topological space $X$. Then we say that $\mu$ is

(i) Zocally bounded if, for every $x \in X$, there is a $V \in V(x)$ such that $\mu(V)<\infty$;

(ii) weakly $\tau$-smooth if $\sup _{\alpha} \mu\left(U_{\alpha}\right)=\mu(X)$ for each net

$$
\left\{U_{\alpha}\right\} \subset O(X) \text { such that } U_{\alpha} \uparrow X \text {; }
$$


(iii) $\tau$-smooth if $\sup _{\alpha} \mu\left(U_{\alpha}\right)=\mu(U)$ for each net

$\left\{U_{\alpha}\right\} \subset O(X)$ such that $U_{\alpha} \uparrow U$.

Note that a finite real-valued $\tau$-smooth Borel measure on a regular space is regular (see Gardner [10, Theorem 5.4]).

DEFINITION 1.2.8. Let $\mu$ be a Borel measure on a topological space $X$. Then $\mu$ is called

(i) a pre-Radon measure if $\mu$ is locally bounded, regular and $\tau$-smooth;

(ii) a Radon measure if $\mu$ is locally bounded, semi-finite and compact regular.

A Radon measure is $\tau$-smooth, and a $\sigma$-finite Radon measure is a preRadon measure. However, all Radon measures are not necessarily pre-Radon measures. There are Radon measures which are not outer regular (see, for example, Gruenhage and Pfeffer [13, Example 7]).

A semi-finite pre-Radon measure on a locally compact space or a complete metric space is a Radon measure. In general, we have

THEOREM 1.2.9 (Amemiya, Okada and Okazaki [2, Theorem 7.4). Let X be a complete regular space such that $X$ is a Borel subset of its StoneČech compactification. Then every semi-finite pre-Radon measure on $X$ is a Radon measure.

Fremlin has given the concept of quasi-Radon measures which are similar to pre-Radon measures. As for its definition, see Fremlin [9, §72]. He has also shown the author the relation between a quasi-Radon measure and preRadon measure as follows.

THEOREM 1.2.10 (Fremlin). (i) Let $\mu$ be a pre-Radon measure on a topological space $X$. Then their exists a unique quasi-Radon measure $v$ on $\left(X, \Sigma_{\mu^{*}}\right)$ such that $\nu(B)=\mu^{*}(B)$ for all $B \in \Sigma_{\mu^{*}}$ with $\mu^{*}(B)<\infty$, where $\Sigma_{\mu^{*}}$ is the family of all $\mu^{*}$-measurable sets. Furthermore, $\nu=\mu$ 
on $O(X)$.

(ii) Let $\nu$ be a locally bounded quasi-Radon measure on $(X, \Sigma)$. Then there exists a unique pre-Radon measure $\mu$ on $X$ such that $\mu=\nu$ on $O(X)$

The support of a Borel measure $\mu$ on a topological space $X$ is

$$
S(\mu)=\{x \in X: \mu(V)>0 \text { for every } V \in V(x)\} .
$$

If $\mu$ is finite, then $S(\mu)=n\{F \in F(X): \mu(F)=\mu(X)\}$, but it does not necessarily hold that $\mu(X)=\mu(S(\mu))$ (see Example 1.2.15).

The following propositions are easily verified from the definitions.

PROPOSITION 1.2.11. Every non-zero weakly $\tau$-smooth Borel measure on a topological space $X$ has a non-empty support. In particular, a non-zero pre-Radon measure as well as a non-zero Radon measure has a non-empty support.

PROPOSITION 1.2.12. Let $\mu$ be a Borel measure on a completely regular space $X$. Then

$$
S^{*}\left(\left.\mu\right|_{B^{*}(X)}\right)=S(\mu)
$$

EXAMPLE 1.2.13. Let $X$ be a topological space. For an $x \in X$, we define a Borel measure $\delta_{x}$ by

$$
\delta_{x}(B)= \begin{cases}1 & \text { if } x \in B, \\ 0 & \text { if } x \notin B .\end{cases}
$$

We call $\delta_{x}$ the Dirac measure at $x$. It is obvious that $\delta_{x}$ is a Radon measure and $S\left(\delta_{x}\right)=\{x\}$, and that $\left.\delta_{x}\right|_{B^{*}(X)}$ is a tight measure on $X$.

EXAMPLE 1.2.14. Let $X$ be a topological space, and let $\left\{x_{n}\right\}_{n \in \mathbb{N}}$ be a sequence in $X$ converging to some $x \in X$ such that $x_{n} \neq x_{m}$ if $n \neq m$. Then a Borel measure $\sum_{n \in \mathbb{N}} n \delta_{x_{n}}$ is not locally bounded although it is o-finite. 
EXAMPLE 1.2.15 (J. Dieudonné). Let $\Omega$ be the first uncountable ordinal and $[0, \Omega]$ the set of ordinals less than or equal to $\Omega$ with the usual interval topology. For simplicity, let $X=[0, \Omega]$. We define a two-valued set function $\mu$ on $B(X)$ by $\mu(B)=1$ or 0 according as a Borel set $B \in B(X)$ does or does not include an unbounded closed subset of $X \backslash\{\Omega\} \quad X$. Then $\mu$ is a Borel measure ( $c f$. Halmos $[16, \S 52(10)]$ and called the Dieudonné measure. It follows from the definition that $\mu(\{\Omega\})=0$, and that $\mu(V)=1$ for every $V \in V(\Omega)$. Then we have $S(\mu)=\{\Omega\}$, and $\mu$ is not regular.

EXAMPLE 1.2.16. Under the same notation as in Example 1.2.15, let $\nu=\mu_{Y}$, where $Y=[0, \Omega)=[0, \Omega]-\{\Omega\}$. Then $\nu$ is a regular Borel. measure by Gruenhage and Pfeffer [13, Example 5], and $S(\nu)=\varnothing$. So $\nu$ is not a weakly $\tau$-smooth Borel measure. It is clear that $\iota(\nu)=\mu$, where $\iota: Y \rightarrow X$ is the natural injection. Furthermore, we can claim that $Y$ is not realcompact. In fact, $\left.\nu\right|_{B^{*}(Y)}$ is a two-valued Baire measure which is not $\tau$-smooth, hence Varadarajan [47, Theorem IV.6] assures us that $Y$ is not realcompact.

All vector spaces dealt with in this thesis are over the real number field $\mathbb{R}$.

Let $X$ be a locally convex space and $X^{\prime}$ its topological dual space. For $F \subset X^{\prime}$, let $F^{1}=\{x \in X:\langle x, \xi\rangle=0$ for all $\xi \in F\}$, and let $\pi_{F}$ denote the natural map from $X$ to $X / F^{2}$. If $F D\left(X^{\prime}\right)$ denotes the family of all finite dimensional subspaces of $X^{\prime}$, then $\pi_{F}^{-1}\left(B\left(X / F^{\perp}\right)\right)$ is a o-algebra on $X$ for every $F \in F D\left(X^{\prime}\right)$, and $Z\left(X, X^{\prime}\right)=\underset{F \in F D\left(X^{\prime}\right)}{U} \pi_{F}^{-\perp}\left(B\left(X / F^{\perp}\right)\right)$ is an algebra on $X$. Let $C\left(X, X^{\prime}\right)=\sigma\left(Z\left(X, X^{\prime}\right)\right)$, and let

us call it the cylindrical $\sigma$-algebra of $X$. A cylinder set-measure $m$ on $X$ is a non-negative, real-valued, finitely additive set function on 
$Z\left(X, X^{\prime}\right)$ such that $m$ is o-additive on $\pi_{F}^{-1}\left(B\left(X / F^{\perp}\right)\right)$ for all $F \in F D\left(X^{\prime}\right)$. A measure on $C\left(X, X^{\prime}\right)$ is called a cylindrical measure. Note that $C\left(X, X^{\prime}\right)$ is the minimal $\sigma$-algebra which makes all functionals in $X^{\prime}$ measurable.

Let $y$ be a linear subspace of $X$ endowed with the relative topology. Then we have $Z\left(Y, Y^{\prime}\right)=Y \cap Z\left(X, X^{\prime}\right)$ and $C\left(Y, Y^{\prime}\right)=Y \cap C\left(X, X^{\prime}\right)$.

In a similar way, we can define $Z\left(X^{\prime}, X\right)$ and $C\left(X^{\prime}, X\right)$.

\section{3. $\sigma$-algebras}

In general, the Baire field $B^{*}(X)$ of a topological space $X$ is a sub- $\sigma$-algebra of the Borel field $B(X)$. Suppose that $X$ is a perfectly normal space; that is, $X$ is normal and each closed subset is a $G_{\delta}$-set. Then $B^{*}(X)=B(X)$. In particular, if $X$ is a metric space, then $B^{*}(X)=B(X)$. As far as the author knows, it is not known whether or not $B^{*}(X)=B(X)$ implies that $X$ is perfectly normal.

There are some topological spaces on which the Baire fields are different from the Borel fields.

PROPOSITION 1.3.1. Under the same notation as in Example 1.2.15, $B^{*}([0, \Omega]) \varsubsetneqq B([0, \Omega])$

Proof. There are two alternative proofs (I) and (II).

(I) Assume that $B^{*}([0, \Omega])=B([0, \Omega])$. Then the Dieudonne measure $\mu$ is a Baire measure, whence it is regular. This is a contradiction.

(II) Assume that $B^{*}([0, \Omega])=B([0, \Omega])$. Then

$[0, \Omega) \in B^{*}([0, \Omega])$; hence $[0, \Omega)$ is realcompact by Moran $[31$, Proposition 2.5]. However, $[0, \Omega)$ is not realcompact as we have shown in Example 1.2.16. //

Let $\left\{X_{\lambda}\right\}_{\lambda \in \Lambda}$ be a family of completely regular spaces. By $\underset{\lambda \in \Lambda}{\otimes} C\left(X_{\lambda}\right)$ 
we denote the subring of $C\left(\prod_{\lambda \in \Lambda} x_{\lambda}\right)$ generated by $\underset{\lambda \in \Lambda}{U}\left\{f \circ p_{\lambda}: f \in C\left(X_{\lambda}\right)\right\}$, where $p_{\lambda}$ is the projection of $\prod_{\lambda \in \Lambda} X_{\lambda}$ to $X_{\lambda}$.

PROPOSITION 1.3.2. Let $\left\{X_{\lambda}\right\}_{\lambda \in \Lambda}$ be a family of completely regular spaces. If, for each countable subset $\Lambda_{0}$ of $\Lambda$, the subproduct space $\prod_{\lambda \in \Lambda_{0}} X_{\lambda}$ is a Lindelöf space, then $\underset{\lambda \in \Lambda}{\otimes} B^{*}\left(X_{\lambda}\right)=B^{*}\left(\prod_{\lambda \in \Lambda} X_{\lambda}\right)$. Proof. It is obvious that $\underset{\lambda \in \Lambda}{\otimes} B^{*}\left(X_{\lambda}\right) \subset B^{*}\left(\prod_{\lambda \in \Lambda} X_{\lambda}\right)$. For each $f \in C\left(\prod_{\lambda \in \Lambda} X_{\lambda}\right)$, there is a sequence $\left\{f_{n}\right\}_{n \in \mathbb{N}} \subset \underset{\lambda \in \Lambda}{\otimes} C\left(X_{\lambda}\right)$ such that $f(x)=\lim _{n \rightarrow \infty} f_{n}(x)$ for all $x \in \prod_{\lambda \in \Lambda} X_{\lambda}$ by Hager [14, Theorem 2.2]. Since every $f_{n}$ is $\underset{\lambda \in \Lambda}{\otimes} B^{*}\left(X_{\lambda}\right)$-measurable, $f$ is also $\underset{\lambda \in \Lambda}{\otimes} B^{*}\left(X_{\lambda}\right)$-measurable, which means that $f^{-1}(0) \in \underset{\lambda \in \Lambda}{\otimes} B^{*}\left(X_{\lambda}\right)$ for each $f \in C\left(\prod_{\lambda \in \Lambda} X_{\lambda}\right)$. Hence $B^{*}\left(\prod_{\lambda \in \Lambda} x_{\lambda}\right) \subset \underset{\lambda \in \Lambda}{\otimes} B^{*}\left(X_{\lambda}\right)$

COROLLARY 1.3.2. Let $\left\{X_{\lambda}\right\}_{\lambda \in \Lambda}$ be a family of compact spaces. Then $\otimes_{\lambda \in \Lambda} B^{*}\left(X_{\lambda}\right)=B *\left(\prod_{\lambda \in \Lambda} X_{\lambda}\right)$

Let $B X$ denote the Stone-Čech compactification of a completely regular space $X$.

COROLLARY 1.3.3. Let $\left\{X_{\lambda}\right\}_{\lambda \in \Lambda}$ be a family of completely regular spaces such that $\prod_{\lambda \in \Lambda} X_{\lambda}$ is pseudo-compact. Then $\underset{\lambda \in \Lambda}{\otimes} B^{*}\left(X_{\lambda}\right)=B^{*}\left(\prod_{\lambda \in \Lambda} X_{\lambda}\right)_{j}$. Proof. By Glicksberg [12, Theorem 1], $\beta \prod_{\lambda \in \Lambda} X_{\lambda}=\prod_{\lambda \in \Lambda} \beta X_{\lambda} \cdot$ It 
follows from Corollary 1.3.2 that $\underset{\lambda \in \Lambda}{\otimes} B^{*}\left(B X_{\lambda}\right)=B^{*}\left(\prod_{\lambda \in \Lambda} B X_{\lambda}\right)$. Then

$$
\begin{aligned}
\otimes_{\lambda \in \Lambda} B^{*}\left(X_{\lambda}\right) & =\left(\otimes_{\lambda \in \Lambda} B^{*}\left(B X_{\lambda}\right)\right) \cap \prod_{\lambda \in \Lambda} x_{\lambda} \\
& =B^{*}\left(\prod_{\lambda \in \Lambda} B X_{\lambda}\right) \cap \prod_{\lambda \in \Lambda} x_{\lambda} \\
& =B^{*}\left(\beta \prod_{\lambda \in \Lambda} x_{\lambda}\right) \cap \prod_{\lambda \in \Lambda} x_{\lambda} \\
& =B^{*}\left(\prod_{\lambda \in \Lambda} x_{\lambda}\right) .
\end{aligned}
$$

PROPOSITION 1.3.4. Let $\left\{X_{\lambda}\right\}_{\lambda \in \Lambda}$ be an uncountable family of nontrivial topological spaces; that is, every $X_{\lambda}$ contains at least two elements. Then

$$
\otimes_{\lambda \in \Lambda} B\left(X_{\lambda}\right) \varsubsetneqq B\left(\prod_{\lambda \in \Lambda} x_{\lambda}\right)
$$

Proof. For each $\lambda \in \Lambda$, there is an $F_{\lambda} \in F\left(X_{\lambda}\right)$ such that $F_{\lambda} \neq \varnothing, X_{\lambda}$. Then $\prod_{\lambda \in \Lambda} F_{\lambda} \notin \underset{\lambda \in \Lambda}{\otimes} B\left(X_{\lambda}\right)$ since

$$
\otimes \underset{\lambda \in \Lambda}{\otimes} B\left(X_{\lambda}\right)=U\left\{(\underbrace{\otimes}_{\lambda \in \Lambda_{0}} B\left(x_{\lambda}\right)) \times \prod_{\lambda \notin \Lambda_{0}} x_{\lambda}: \Lambda_{0} \text { is a countable subset of } \Lambda\right\} \text {. }
$$

However, $\prod_{\lambda \in \Lambda} F_{\lambda} \in B\left(\prod_{\lambda \in \Lambda} X_{\lambda}\right)$ since $\prod_{\lambda \in \Lambda} F_{\lambda}$ is closed. //

COROLLARY 1.3.5. Let $\left\{X_{\lambda}\right\}_{\lambda \in \Lambda}$ be a family of non-trivial topological spaces which satisfy the second axiom of countability. Then

$$
B *\left(\prod_{\lambda \in \Lambda} x_{\lambda}\right) \varsubsetneqq B\left(\prod_{\lambda \in \Lambda} x_{\lambda}\right) \text {. }
$$

Proof. It follows from Proposition 1.3.2 and Proposition 1.3.4 that

$$
B^{*}\left(\prod_{\lambda \in \Lambda} X_{\lambda}\right)=\underset{\lambda \in \Lambda}{\otimes} B^{*}\left(X_{\lambda}\right) \subset \underset{\lambda \in \Lambda}{\otimes} B\left(X_{\lambda}\right) \varsubsetneqq B\left(\prod_{\lambda \in \Lambda} X_{\lambda}\right) \cdot \quad / /
$$

Similarly we have 
COROLLARY 1.3.6. Suppose that $\left\{X_{\lambda}\right\}_{\lambda \in \Lambda}$ is a family of non-trivial compact spaces. Then

$$
B *\left(\prod_{\lambda \in \Lambda} x_{\lambda}\right) \varsubsetneqq B\left(\prod_{\lambda \in \Lambda} x_{\lambda}\right)
$$

Now we concentrate on locally convex spaces. Let $X$ be a locally convex space. By Edgar [8, Theorem 2.3] we have $C\left(X, X^{\prime}\right)=B^{*}\left(X_{\sigma\left(X, X^{\prime}\right)}\right)$, where $\sigma\left(X, X^{\prime}\right)$ denotes the weak topology on $X$. Thus, in general, we have the following relationships:

(I) $C\left(X, X^{\prime}\right) \subset B\left(X_{\sigma\left(X, X^{\prime}\right)}\right) \subset B(X)$; and

(II) $C\left(X, X^{\prime}\right) \subset B^{*}(X) \subset B(X)$.

PROPOSITION 1.3.7. Let $X$ be a locally convex space. If $C\left(X, X^{\prime}\right) \rightarrow\{0\}$, then the cardinal number of $X$ is equal to that of $\mathbb{R}$.

Proof. Let $f: X \times X \rightarrow X$ be defined by $f(x, y)=x-y$. Then $f$ is $\left(C\left(X, X^{\prime}\right) \otimes C\left(X, X^{\prime}\right)-C\left(X, X^{\prime}\right)\right)$-measurable. Since $\{0\} \in C\left(X, X^{\prime}\right)$, $\{(x, x) \in X \times X: x \in X\}=f^{-1}(0) \in C\left(X, X^{\prime}\right)$. By Hoffmann-Jфrgensen [19, Theorem $\left[\right.$.3.5] there is an injection from $X$ into $\mathbb{R}$ which is $C\left(X, X^{\prime}\right)$ measurable. This completes the proof. //

COROLLARY 1.3.8. If the cardinal number of a locally convex space $X$ is greater than that of $\mathbb{R}$, then

$$
C\left(X, X^{\prime}\right)=B^{*}\left(X_{\sigma\left(X, X^{\prime}\right)}\right) \varsubsetneqq B\left(X_{\sigma\left(X, X^{\prime}\right)}\right) \subset B(X) .
$$

Recall that a topological space is called hereditarily Lindelöf if its every subspace is Lindelöf.

THEOREM 1.3.9 (Mitoma, Okada and Okazaki $[28$, Theorem 3.6]). Let $X$ be a hereditarily Lindelöf locally convex space. Then

$$
C\left(X, X^{\prime}\right)=B\left(X_{\sigma\left(X, X^{\prime}\right)}\right)=B(X) .
$$

COROLLARY 1.3.10. Suppose that $X$ is a separable Frechet space. Then $C\left(X, X^{\prime}\right)=B\left(X_{\sigma\left(X, X^{\prime}\right)}\right)=B(X)$. 
Let $\left\{X_{\lambda}\right\}_{\lambda \in \Lambda}$ be a family of locally convex spaces. Then it follows

that

$$
\otimes_{\lambda \in \Lambda} C\left(X_{\lambda}, X_{\lambda}^{\prime}\right)=C\left(\prod_{\lambda \in \Lambda} x_{\lambda},\left(\prod_{\lambda \in \Lambda} x_{\lambda}\right)^{\prime}\right) .
$$

The following theorem is shown by Okazaki.

THEOREM 1.3.11. If $X$ is a Lindelöf Zocally convex space, then $C\left(X, X^{\prime}\right)=B^{*}(X)$

Proof. Let $\left\{q_{\lambda}\right\}_{\lambda \in \Lambda}$ be the family of continuous seminorms which determines the locally convex topology on $X$. Then we can regard $X$ as a subspace of $X_{0}=\prod_{\lambda \in \Lambda} X_{\lambda}$, where $X_{\lambda}=X /$ ker $q_{\lambda}$. Then $X$ is a Baireembedding subspace since it is a Lindelöf space. Since every $X_{\lambda}$ is a separable normed space, $C\left(X_{\lambda}, X_{\lambda}^{\prime}\right)=B^{*}\left(X_{\lambda}\right)=B\left(X_{\lambda}\right)$ by Theorem 1.3.9. Thus we have

$$
\begin{aligned}
C\left(X, X^{\prime}\right) & =X \cap C\left(X_{0}, X_{0}^{\prime}\right)=X \cap\left(\otimes_{\lambda \in \Lambda}^{\otimes} C\left(X_{\lambda}, X_{\lambda}^{\prime}\right)\right) \\
& =X \cap\left(\otimes_{\lambda \in \Lambda}^{\otimes} B^{*}\left(X_{\lambda}\right)\right)=X \cap B^{*}\left(X_{0}\right) \\
& =B^{*}(X) .
\end{aligned}
$$

A norm \|\| on a Banach space $X$ is said to be a Kadec norm if the weak and the strong topologies of $X$ agree on $\{x \in X:\|x\|=I\}$. It is known that if $(X,\|\|)$ is a locally uniformly convex Banach space, then the norm \|\| is a Kadec norm.

THEOREM 1.3.12 (Edgar [8, Theorem 1.1]). Let $X$ be a Banach space which admits a Kadec norm; that is, there is a Kadec norm equivalent to the original norm of $X$. Then $B\left(X_{\sigma\left(X, X^{\prime}\right)}\right)=B(X)$.

By Edgar [8, Proposition 2.5], there are a Banach space $X$ and a closed $G_{\delta}$-subset $A$ of $X_{\sigma\left(X, X^{\prime}\right)}$ such that $A \notin B^{*}\left(X_{\sigma\left(X, X^{\prime}\right)}\right)=C\left(X, X^{\prime}\right)$. In 
particular, $X_{\sigma\left(X, X^{\prime}\right)}$ is not perfectly normal.

Masani [26, Theorem 3.13] has shown the following theorem.

THEOREM 1.3.13. Let $X$ be a Hilbert space such that $C\left(X, X^{\prime}\right)=B(X)$. Then $X$ is separable. 


\section{CHAPTER II}

\section{EXTENSION THEOREMS}

\subsection{Introduction}

In $\S 2.2$, we prove pre-Radon extension theorems; that is, we examine necessary and sufficient conditions for a set function defined on a ring or an algebra of subsets of a topological space $X$ to be extendible to a pre-Radon measure on $X$.

The following three theorems give sufficient conditions for a Baire measure to be extendible to a Borel measure.

THEOREM 2.1.1 (Mařík [25]). Let $X$ be a normal, countably paracompact space. Then every Baire measure on $X$ can be extended to a unique Borel measure on $X$.

For the definition of countably paracompact spaces, see $\$ 5.1$.

THEOREM 2.1.2 (Knowles [23, Theorem 2.4], Kirk [22, Theorem 1.14]). Let $\mu$ be a $\tau$-smooth Baire measure on a completely regular topological space $X$; then $\mu$ is extendible to a unique $\tau$-smooth Borel measure on $X$.

THEOREM 2.1.3 (Knowles [23, Theorem 2.5]). If $\mu$ is a tight Baire measure on a completely regular topological space, then $\mu$ is extendible to a unique Radon measure on $X$.

In $\$ 2.3$, we deal with Kolmogorov's extension theorem for infinite Radon measures. Yamasahi [49] has already studied the extension problem of a self-consistent system of infinite measures on the assumption that every self-consistent system of probability measures has a unique $\sigma$-additive extension.

Let $I$ be a directed set and $\left\{F_{L}\right\}_{\iota \in I}$ a family of topological spaces such that, for each pair $(\iota, k)$ of indices with $\iota \leq k$, there is a 
continuous map $f_{\iota K}: F_{K} \rightarrow F_{\mathcal{L}}$ and that these maps satisfy the following:

(i) $f_{\iota \iota}$ is the identity map of $F_{\iota}$ for each $\iota \in I$;

(ii) $f_{\iota \lambda}=f_{\iota k} \circ f_{K \lambda}$ for $\iota \leq k \leq \lambda$.

Then we call this system $\left\{\left(F_{\imath}, f_{\iota K}\right): \iota \leq K, \iota, k \in I\right\}$ a projective system of topological spaces. Let $\lim F_{\iota}$ denote its projective limit; that is,

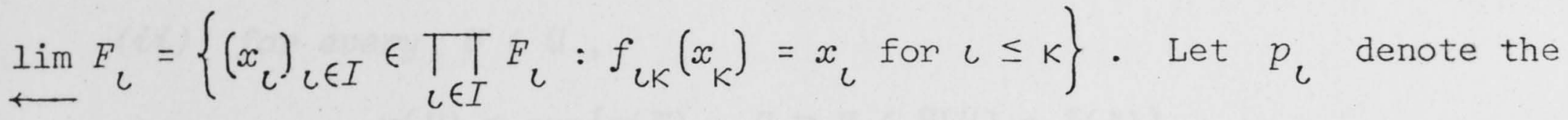
projection of $\lim F_{\iota}$ to $F_{\iota} ;$ then we have $p_{\iota}=f_{\iota K} \circ p_{K}$ on $\lim F_{\iota}$. Let $D(I)=\left\{\left(\iota_{n}\right)_{n \in \mathbb{N}}: \iota_{1} \leq \iota_{2} \leq \ldots, \iota_{n} \in I\right\}$. For $M=\left(\iota_{n}\right)_{n \in \mathbb{N}} \in D(I)$, let $p_{M}$ denote the natural projection of $F=\lim F_{c}$ to $F_{M}=\lim F_{c_{n}}$. We say that the projective system $\left\{\left(F_{\iota}, P_{\iota K}\right): \iota \leq K, \iota, K \in I\right\}$ is sequentially maximal if $p_{M}$ is surjective for all $M \in D(I)$.

Let $\mu_{\iota}(\iota \in I)$ be a Borel measure on $F_{\iota}$. We call $\left\{\mu_{\iota}\right\}_{\iota \in I}$ selfconsistent if $f_{\iota K}\left(\mu_{K}\right)=\mu_{\iota}$ for each pair $(\iota, \kappa)$ with $\iota \leq k$. The Kolmogorov's extension problem is whether there exists a $\sigma$-additive measure $\mu$ on the $\sigma$-algebra $\sigma\left(\cup_{\iota \in I}^{U} p_{l}^{-I}\left(B\left(F_{l}\right)\right)\right)$ such that $p_{\ell}(\mu)=\mu_{\iota}$. Bochner has proved the following theorem.

THEOREM 2.1.5 (Bochner [4, Theorem 5.1.1]). Let $F=\lim F_{\imath}$ be the projective limit of a sequentially maximal projective system $\left\{\left(F_{\imath}, p_{L K}\right): \iota \leq K, \iota, k \in I\right\}$ such that $p_{\iota}$ is surjective for all $\iota \in I$, and let $\left\{\mu_{\iota}\right\}_{\iota \in I}$ be a self-consistent system of Radon probability measures on $\left\{F_{\iota}\right\}_{\imath \in I}$. Then there exists a unique o-additive measure $\mu$ on $\left(F, \underset{M \in D(I)}{U} p_{M}^{-1}\left(B\left(F_{M}\right)\right)\right)$ such that $p_{\iota}(\mu)=\mu_{\iota}$ on $F_{\iota}$ for every $\iota \in I$. 


\subsection{Pre-Radon Extension}

THEOREM 2.2.1. Let $X$ be a topological space, $U$ a paving generated by an open base of $X$, and $m$ a non-negative, real-valued, finitely additive set function on $\mathrm{R}[\mathrm{U}]$. Then $m$ is extendible to a unique preRadon measure on $X$ if and only if $m$ satisfies the following conditions:

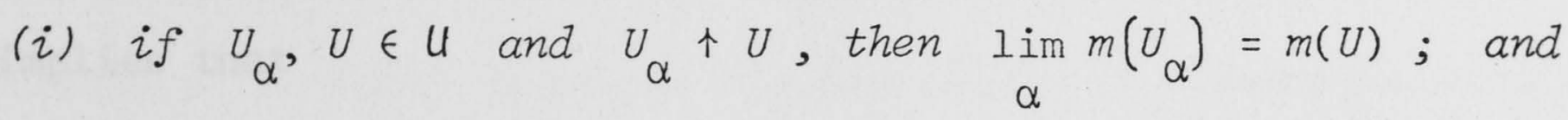

(ii) for every $U \in U$,

$$
m(U)=\sup \{m(F): U \supset F \in R[U] \cap F(X)\} .
$$

Proof. It is sufficient to prove the 'if' portion. Suppose that, for two nets $\left\{U_{\alpha}\right\},\left\{V_{\beta}\right\} \subset U, U_{\alpha} \uparrow 0$ and $V_{\beta} \uparrow 0$; then we have

$$
\lim _{\alpha} m\left(U_{\alpha}\right)=\lim _{\beta} m\left(V_{\beta}\right)
$$

For $0 \in O(X)$, let

$$
\lambda(0)=\sup \{m(U): 0 \supset U \in U\} .
$$

Then $\lambda$ is an increasing, non-negative, subadditive set function on $O(X)$. If $o_{\alpha} \in O(X)$ and $o_{\alpha} \uparrow 0$, then we have $\lim _{\alpha} \lambda\left(O_{\alpha}\right)=\lambda(0)$. We define a set function $\lambda^{0}$ by

$$
\lambda^{0}(A)=\inf \{\lambda(0): A \subset 0 \in O(X)\}
$$

for every subset $A$ of $X$. This $\lambda^{0}$ is an outer measure defined on the class of all subsets of $X$. Now we show that each open set $0 \in O(X)$ is $\lambda^{0}$-measurable. It is sufficient to show that

$$
\lambda^{0}(A) \geq \lambda^{0}(A \cap 0)+\lambda^{0}(A-O)
$$

for each subset $A$ of $X$ with $\lambda^{0}(A)<\infty$. Given $\varepsilon>0$, there is an $O_{1} \in O(X)$ such that $O_{1} \supset A$ and $\lambda\left(O_{1}\right)<\varepsilon+\lambda^{O}(A)$. We can take a net $\left\{U_{\alpha}\right\} \subset U$ such that $U_{\alpha} \uparrow O_{1}$. For every $V \in U$ with $V \subset 0$, there is an $F \in R[U] \cap F(X)$ such that $F \subset V$ and $m(F)+\varepsilon>m(V)$ by $(i i)$. Then it 
follows that

$$
m\left(U_{\alpha}-F\right)-m\left(U_{\alpha}-V\right) \leq m(V-F)<\varepsilon
$$

for all $\alpha$. Hence we have

$$
\varepsilon+\lim _{\alpha} m\left(U_{\alpha}-V\right)>\lim _{\alpha} m\left(U_{\alpha}-F\right)=\lambda\left(O_{1}-F\right) \geq \lambda^{0}(A-O)
$$

which implies that

$$
\begin{array}{r}
\varepsilon+\lambda^{0}(A)>\lambda\left(O_{1}\right)=\lim _{\alpha} m\left(U_{\alpha}\right)=\lim _{\alpha}\left(m\left(U_{\alpha} \cap V\right)+m\left(U_{\alpha}-V\right)\right) \\
>\lambda\left(O_{I} \cap V\right)+\lambda^{0}(A-O)-\varepsilon .
\end{array}
$$

Since $V$ is arbitrary, we have

$$
\begin{aligned}
2 \varepsilon+\lambda^{0}(A) & \geq \lambda\left(O_{1} \cap 0\right)+\lambda^{0}(A-0) \\
& \geq \lambda^{0}(A \cap 0)+\lambda^{0}(A-O)
\end{aligned}
$$

so that 0 is $\lambda^{0}$-measurable. Thus every Borel subset of $X$ is $\lambda^{0}$-measurable; that is, $\mu=\left.\lambda^{0}\right|_{B(X)}$ is a Borel measure. It follows from the construction that $\mu$ is a pre-Radon measure.

Next we prove that $\mu$ is an extension of $m$. For every $A \in R[U]$, there are $w_{i}, V_{i} \in U(i=1,2, \ldots, m)$ which satisfy $(i)$, (ii) and (iii) in Lemma 1.2.1. Hence we have

$$
\begin{aligned}
m(A) & \left.=m \sum_{i=1}^{n}\left(W_{i}-V_{i}\right)\right) \\
& =\sum_{i=1}^{n}\left(m\left(W_{i}\right)-m\left(V_{i}\right)\right) \\
& =\sum_{i=1}^{n}\left(\mu\left(w_{i}\right)-\mu\left(V_{i}\right)\right)=\mu(A) .
\end{aligned}
$$

Finally we have to prove the uniqueness of $\mu$. Let $\nu$ be another pre-Radon measure such that $\nu=m$ on $R[U]$. For every $O \in O(X)$, there is a net $\left\{U_{\alpha}\right\} \subset U$ such that $U_{\alpha} \uparrow 0$, then we have 


$$
\mu(0)=\lim _{\alpha} \mu\left(U_{\alpha}\right)=\lim _{\alpha} m\left(U_{\alpha}\right)=\lim _{\alpha} \nu\left(U_{\alpha}\right)=v(0)
$$

Since both $\mu$ and $\nu$ are outer regular, $\mu=\nu . \quad / /$

Next we consider the algebra $\mathrm{A}[\mathrm{U}]$.

THEOREM 2.2.2. Let $X$ be a topological space, $U$ a paving generated by an open base of $X$. Then a non-negative, extended real-valued, o-additive set function $m$ on the algebra $A[U]$ is extendible to a unique $\sigma$-finite pre-Radon measure on. $X$ if and only if $m$ satisfies the following conditions:

(i) there exists an increasing sequence $\left\{U_{n}\right\}_{n \in \mathbb{N}} \subset U$ such

$$
\text { that } X=\bigcup_{n \in \mathbb{N}} U_{n} \text { and } m\left(U_{n}\right)<\infty \text { for each } n \in \mathbb{N} \text {; }
$$

(ii) if $U_{\alpha}, U \in U, U_{\alpha} \uparrow U$ and $m(U)<\infty$, then

$$
\lim _{\alpha} m\left(U_{\alpha}\right)=m(U)
$$

(iii) for each $U \in U$ such that $m(U)<\infty$,

$$
m(U)=\sup \{m(F): U \supset F \in A[U] \cap F(X)\} .
$$

Proof. It suffices to show the 'if' part. For $0 \in O(X)$, we let

$$
\lambda(0)=\sup \{m(U): 0 \supset U \in U \text { and } m(U)<\infty\} \text {, }
$$

and let

$$
\lambda^{0}(0)=\inf \{\lambda(0): A \subset 0 \in O(X)\} .
$$

Then by the same manner as in the proof of Theorem 2.2.1 every open set is $\lambda^{0}$-measurable, and $\mu=\left.\lambda^{0}\right|_{B(X)}$ is a $\sigma$-finite pre-Radon measure.

Now we prove that $\mu$ is an extension of $m$. For every $U \in \dot{u}$, the algebra $U \cap A[U]$ is generated by $U \cap U$. Then, for every $A \in A[U]$, there are $v_{i}, w_{i} \in U(i=1,2, \ldots, n)$ satisfying $(i),(i i)$ and $(i i i)$ in Lemma 2.1.1 such that $A \cap U=\bigcup_{i=1}^{n}\left(U \cap W_{i}-U \cap V_{i}\right)$. Hence we have $m(U \cap A)=\mu(U \cap A)$ if $m(U)<\infty$. It follows that 


$$
m(A)=\lim _{n \rightarrow \infty} m\left(U_{n} \cap A\right)=\lim _{n \rightarrow \infty} \mu\left(U_{n} \cap A\right)=\mu(A)
$$

Consequently, $\mu$ is an extension of $m$.

The uniqueness of $\mu$ is proved by the same way as in the proof of Theorem 2.2.1. //

COROLLARY 2.2.3. Let $X$ be a topological space and $U$ a paving generated by an open base such that $X \in U$, and let $m$ be a non-negative, finite real-valued, finitely additive set function on the algebra $A[U]$. Then $m$ is extendible to a unique pre-Radon measure on $X$ if and only if $m$ satisfies the following:

(i) for $U_{\alpha} \in U$ such that $U_{\alpha} \uparrow X, \underset{\alpha}{\lim m\left(U_{\alpha}\right)=m(X) ; \text { and }}$

(ii) for alz $U \in U$,

$$
m(U)=\sup \{m(F): U \supset F \in A[U] \cap F(X)\} .
$$

Let us note that Theorem 2.1.2 can be a corollary of Corollary 2.2.3.

COROLLARY 2.2.4. Let $X$ be a topological space and $U$ a paving generated by an open base of $X$. A real-valued finitely additive set function $m$ on the algebra $\mathrm{A}[\mathrm{U}]$ can be extended to a unique Radon measure on $X$ if and only if $m$ satisfies the following conditions:

(i) for each $U \in U$,

$$
m(U)=\sup \{m(F): U \supset F \in A[U] \cap F(X)\} ;
$$

and

(ii) $m(X)=\sup \left\{m^{0}(K): K \in K(X)\right\}$, where

$$
m^{0}(K)=\inf \{m(U): K \subset U \in U\} .
$$

Proof. It is sufficient if we prove the 'if' portion. By Corollary 2.2.3, $m$ can be extended to a unique pre-Radon measure $\mu$ on $X$. Given $\varepsilon>0$, there is a $K \in K(X)$ such that $m^{0}(K)>m(X)-\varepsilon$. Since $\mu$ is outer regular, there is an $O \in O(X)$ such that $O \supset K$ and $\mu(K)>\mu(O)-\varepsilon$. We can take a $U \in U$ such that $K \subset U \subset 0$, which implies that 


$$
\mu(K)>\mu(0)-\varepsilon>\mu(U)-\varepsilon \geq m^{0}(K)-\varepsilon \geq \mu(X)-2 \varepsilon .
$$

Hence we have

$$
\text { - } \mu(X)=\sup \{\mu(K): K \in K(X)\} \text {. }
$$

Since $\mu$ is inner regular, $\mu$ is a Radon measure on $X$. //

Remark 2.2.5. In Corollary 2.2.4, it holds that

$$
\mu(K)=m^{0}(K)=\inf \{m(A): K \subset A \in A[U]\}
$$

for all $K \in K(X)$.

Note that Theorem 2.1 .3 can be a corollary of Corollary 2.2.4.

\subsection{Kolmogorov's Extension Theorem}

We begin with the countable case.

THEOREM 2.3.1. Let $T=\lim _{n}$ be the projective limit of a projective system of topological spaces $\left\{\left(T, p_{n m}\right): n \leq m, n, m \in \mathbb{N}\right\}$, and Let $\left\{\mu_{n}\right\}_{n \in \mathbb{N}}$ be a self-consistent family of Radon measures on $\left\{T_{n}\right\}_{n \in \mathbb{N}}$. Then there exists a unique Radon measure $\mu$ on $T$ such that $p_{n}(\mu)=\mu_{n}$, where $p_{n}$ is the projection of $T$ to $T_{n}$.

Proof. Let $\left\{U_{\lambda}\right\}_{\lambda \in \Lambda}$ be the family of open subsets of $T_{1}$ such that $\mu_{1}\left(U_{\lambda}\right)<\infty$. We may assume that $\Lambda$ is directed. Fixed $\lambda \in \Lambda$, $\left\{\left(p_{I n}^{-1}\left(U_{\lambda}\right), q_{n m}\right): n \leq m\right\}$ is a projective system of topological spaces, where $q_{n m}=\left.p_{n m}\right|_{p_{I m}^{-1}\left(U_{\lambda}\right)}$. Let $\nu_{\lambda}^{n}=\left.\mu_{n}\right|_{p_{I n}^{-1}\left(U_{\lambda}\right)}$. Note that the projective limit $V_{\lambda}=\lim _{\leftarrow}^{-1}\left(U_{\lambda}\right)$ of $\left\{\left(p_{\ln }^{-1}\left(U_{\lambda}\right), q_{n m}\right): n \leq m\right\}$ is equal to $p_{I}^{-1}\left(U_{\lambda}\right)$. By Bourbaki [5, Théorème 4.2], there is a Radon measure $\nu_{\lambda}$ on $V_{\lambda}$ such that $\left(p_{n} \mid V_{\lambda}\right)\left(\nu_{\lambda}\right)=\nu_{\lambda}^{n}$. Let 


$$
\mu(E)=\sup _{\lambda \in \Lambda} v_{\lambda}\left(E \cap V_{\lambda}\right)
$$

for every $E \in B(T)$. Since $\left.\mu_{n}\right|_{E_{n}}$ is a Radon measure for all $E_{n} \in B\left(T_{n}\right)$, it follows that

$$
\begin{aligned}
p_{n}(\mu)\left(E_{n}\right) & =\mu\left(p_{n}^{-1}\left(E_{n}\right)\right)=\sup _{\lambda \in \Lambda} \nu_{\lambda}\left(p_{n}^{-1}\left(E_{n}\right) \cap V_{\lambda}\right) \\
& =\sup _{\lambda \in \Lambda} \nu_{\lambda}\left(p_{n}^{-1}\left(E_{n} \cap p_{I n}^{-1}\left(U_{\lambda}\right)\right)\right] \\
& =\sup _{\lambda \in \Lambda} \nu_{\lambda}^{n}\left(E_{n} \cap p_{I n}^{-1}\left(U_{\lambda}\right)\right) \\
& =\sup _{\lambda \in \Lambda} \mu_{n}\left(E_{n} \cap p_{I n}^{-1}\left(U_{\lambda}\right)\right) \\
& =\sup _{\lambda \in \Lambda}\left(\left.\mu_{n}\right|_{E_{n}}\right)\left(E_{n} \cap p_{I n}^{-1}\left(U_{\lambda}\right)\right)=\mu_{n}\left(E_{n}\right) .
\end{aligned}
$$

Hence $p_{n}(\mu)=\mu_{n}$ for every $n \in \mathbb{N}$, which implies that $\mu$ is locally bounded. Since every $\nu_{\lambda}$ is a Radon measure, $\mu$ is a Radon measure on $T$.

Assume that $\nu$ is another Radon measure on $T$ such that $p_{n}(\nu)=\mu_{n}$. For each $K \in K(T)$, we have $K=\bigcap_{n \in \mathbb{N}} p_{n}^{-1} p_{n}\left(K_{n}\right)$ by Bourbaki [5, Proposition 4.2]. Then

$$
\nu(K)=\lim _{n \rightarrow \infty} \nu\left(p_{n}^{-1} p_{n}(K)\right)=\lim _{n \rightarrow \infty} \mu_{n}\left(p_{n}(K)\right)=\mu(K)
$$

that is, $\nu=\mu$. //

Note that if $\mu_{n}$ is $\sigma$-finite for some $n \in \mathbb{N}$, then $\mu$ is $\sigma$-finite and outer regular by Theorem 1.2.4.

Now we show the general case.

THEOREM 2.3.2. Let $T=\lim T_{i}$ be the projective limit of a sequentially maximal projective system of topological spaces 
$\left\{\left(T_{\iota}, p_{\iota K}\right): \iota \leq K, \iota, K \in I\right\}$ such that the projection $p_{\iota}$ of $T^{T}$ to ${ }^{T}{ }_{l}$ is surjective for all $\iota \in I$. If $\left\{\mu_{\imath}\right\}_{\iota \in I}$ is a self-consistent family of Radon measures on $\left\{{ }_{L}\right\}_{\iota} \in I$, then there exists a unique $\sigma$-additive measure on the $\sigma$-algebra $B_{I}=\underset{M \in D(I)}{U} p_{M}^{-1}\left(B\left(T_{M}\right)\right)$ such that $p_{M}(\mu)$ is a Radon measure on $T_{M}$ and $p_{\imath}(\mu)=\mu_{\imath}$ on $T_{\imath}$ for each $\iota \in I$.

Proof. We define a finitely additive set function $\rho$ on the algebra $A=U_{\iota \in I} p_{\iota}^{-1}\left(B\left(T_{\iota}\right)\right)$ by

$$
\rho\left(p_{\iota}^{-1}\left(E_{\iota}\right)\right)=\mu_{\iota}\left(E_{\iota}\right)
$$

for each $p_{\iota}^{-1}\left(E_{\iota}\right) \in A$. This $\rho$ is well-defined since $p_{\iota}$ is surjective. For each $M=\left(\iota_{n}\right)_{n \in \mathbb{N}} \in D(I)$, we denote by $q_{n M}$ the projection of $T_{M}$ to ${ }^{T} \imath_{n}$. Since $\left\{\mu_{\iota_{n}}\right\}_{n \in \mathbb{N}}$ is a self-consistent family of Radon measures on

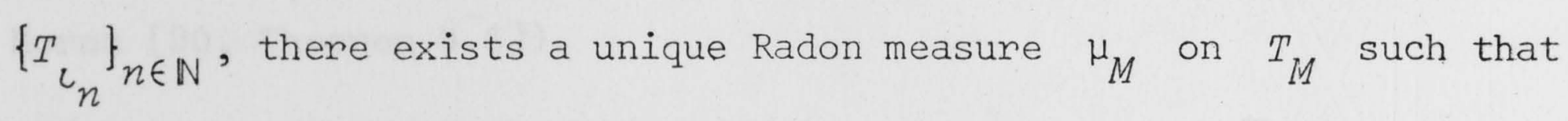
$q_{n M}\left(\mu_{M}\right)=\mu_{c_{n}}$ for every $n \in \mathbb{N}$. We introduce an order relation in $D(I)$ as follows: for $M=\left(\iota_{n}\right)_{n \in \mathbb{N}}, \quad N=\left(k_{n}\right)_{n \in \mathbb{N}} \in \mathcal{D}(I)$,

$$
M \leq N \text { if and only if } \iota_{n} \leq \kappa_{n} \text { for every } n \in \mathbb{N} .
$$

If $M \leq N$, then we let $p_{M N}\left(\left(x_{\kappa_{n}}\right)\right)=\left(p_{\iota_{n}{ }_{n}}\left(x_{\kappa_{n}}\right)\right)$. So we have

$$
p_{M}=p_{M N} \circ P_{N} \text { and } p_{M N}\left(\mu_{N}\right)=\mu_{M} \text { since }
$$

$$
q_{n M} \circ p_{M N}\left(\mu_{N N}\right)=p_{c_{n}{ }^{\kappa} n} \circ q_{n N}\left(\mu_{N N}\right)=p_{c_{n}{ }^{k} n}\left(\mu_{\kappa_{n}}\right)=\mu_{c_{n}} .
$$

Define a measure $\mu$ on $B_{1}$ as follows:

$$
\mu\left(p_{M}^{-1}\left(E_{M}\right)\right)=\mu_{M}\left(E_{M}\right)
$$

for all $p_{M}^{-1}\left(E_{M}\right) \in B_{1}$. It is obvious that $\mu$ is $\sigma$-additive on $B_{\perp}$ and 
that $p_{M}(\mu)=\mu_{M}$ for every $M \in D(I)$.

For each $\iota \in I$, there is an $M=\left(\iota_{n}\right)_{n \in \mathbb{N}} \in D(I)$ such that $\iota_{I}=\iota$; hence $p_{\iota}(\mu)=q_{I M} p_{M}(\mu)=q_{I M}\left(\mu_{M}\right)=\mu_{\iota_{1}}=\mu_{\iota}$. Since $B_{I}$ is a $\sigma$-algebra including $A, \mu$ is a $\sigma$-additive extension of $\rho$.

Suppose that $\nu$ is another $\sigma$-additive extension of $\rho$ on $B_{1}$ such that $p_{M}(\nu)$ is a Radon measure on $T_{M}$ for each $M=\left(\iota_{n}\right)_{n \in \mathbb{N}} \in D(I)$. Then it follows that

$$
q_{n M}\left(p_{M}(\nu)\right)=p_{\iota_{n}}(\nu)=p_{\tau_{n}}(\rho)=p_{\iota_{n}}(\mu)=q_{n M}\left(p_{M}(\mu)\right)
$$

on $T_{c_{n}}$ for every $n \in \mathbb{N}$. Theorem 2.3.1 implies that $p_{M}(\nu)=p_{M}(\mu)$. It follows from the definition of $B_{1}$ that $\nu=\mu \cdot \quad / /$

Remark 2.3.3. In Theorem 2.3.2, $\mu$ is not necessarily extendible to a $\tau$-smooth Borel measure even if every $\mu_{\imath}$ is a probability measure (see Moran [30, Theorem 4.6]). 


\section{CHAPTER III}

\section{APPLICATIONS OF PRE-RADON EXTENSION THEOREMS}

\subsection{Introduction}

In $\S 3.2$, we show that the product of two $\sigma$-finite pre-Radon measures can be extended to a unique pre-Radon measure; and we prove Fubini's theorem with respect to $\sigma$-finite pre-Radon measures.

In $\$ 3.3$, we prove that the infinite product of pre-Radon probability measures is extendible to a unique pre-Radon probability measure. In the countable product case, Tortrat [45, Théorème 2] has given it. However, there seems to be some gap in his proof. So we prove the countable product case by using Fubini's theorem obtained in $\$ 3.2$. The uncountable product case is derived from the countable product case. Moreover, we present a necessary and sufficient condition for the extended pre-Radon measure to be a Radon measure. As an application, we have a pre-Radon gaussian measure which is not a Radon measure (Example 3.3.5).

In $\$ 3.4$, some cylindrical measures are extended to pre-Radon or Radon measures. All cylindrical measures on $\left(X, C\left(X, X^{\prime}\right)\right)$ which can be extended to the Borel field $B(X)$ have not always unique extensions (Example 3.4 .5 ). Schachermayer [42] has given a cylindrical measure which can not be extended to a pre-Radon measure (Example 3.4.6).

\subsection{Fubin's Theorem}

The product of two finite pre-Radon measures is extended to a unique pre-Radon measure as follows.

THEOREM 3.2.1. Let $\mu, \nu$ be finite pre-Radon measures on topological spaces $X, Y$ respectively. Then the product measure $\rho=\mu \otimes \nu$ on $(X \times Y, B(X) \otimes B(Y))$ is extendible to a unique pre-Radon measure $\bar{\rho}$ on the 
product space $X \times Y$.

Proof. Let $u$ be the paving generated by

$$
u_{0}=\{U \times V: U \in O(X), V \in O(Y)\} .
$$

Suppose that $\left\{W_{\alpha}\right\} \subset U, W_{\alpha} \uparrow X$. Let $\left\{U_{\gamma} \times V_{\gamma}: \gamma \in \Gamma\right\}$ be a subfamily of $U_{0}$ such that each $U_{\gamma} \times V_{\gamma}$ is included in some $W_{\alpha}$. It is obvious that $\underset{\gamma \in \Gamma}{U}\left(U_{\gamma} \times V_{\gamma}\right)=X \times Y$. For each $x \in X, \underset{U_{\gamma} \ni x}{U} V_{\gamma}=Y$. Given $\varepsilon>0$, there are $\gamma_{1}^{x}, \gamma_{2}^{x}, \ldots, \gamma_{n(x)}^{x} \in \Gamma$ such that $\nu\left(\begin{array}{cc}n(x) & \\ \underset{i=1}{U} & \gamma_{i}^{x}\end{array}\right)>v(y)-\varepsilon$ since $\nu$ is a pre-Radon measure. Let $U(x)=\prod_{i=1}^{n(x)} \gamma_{i}^{x}$; then there exist $x_{1}, x_{2}, \ldots, x_{k} \in X$ such that $\mu\left(\bigcup_{j=1}^{k} U\left(x_{j}\right)\right)>\mu(X)-\varepsilon$. It follows from the definition of product measures that

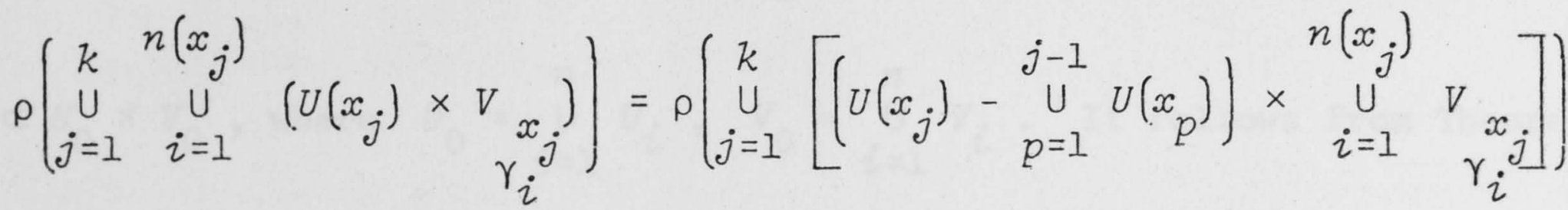

$$
\begin{aligned}
& =\sum_{j=1}^{k} \rho\left(\left(U\left(x_{j}\right)-\bigcup_{p=1}^{j-1} U\left(x_{p}\right)\right) \times \underset{i=1}{U_{j}\left(x_{j}\right)} V_{\gamma_{i}}^{x_{j}}\right) \\
& >\sum_{j=1}^{k} \mu\left(U\left(x_{j}\right)-\bigcup_{p=1}^{j-1} U\left(x_{p}\right)\right)(\nu(Y)-\varepsilon) \\
& =\mu\left(\bigcup_{j=1}^{k} U\left(x_{j}\right)\right)(\nu(Y)-\varepsilon)>(\mu(X)-\varepsilon)(\nu(Y)-\varepsilon) \text {. }
\end{aligned}
$$

Since $\left\{W_{\alpha}\right\}$ is directed, there is an $\alpha_{0}$ such that

$$
\bigcup_{j=1}^{k} \bigcup_{i=1}^{n\left(x_{j}\right)}\left(U\left(x_{j}\right) \times v_{\gamma_{i}}\right) \subset w_{\alpha_{0}}
$$


which implies that $\lim _{\alpha} \rho\left(W_{\alpha}\right)=\rho(X \times Y)$. It follows from Corollary 2.2.3 that the restriction $\left.\rho\right|_{A[U]}$ is extendible to a unique pre-Radon measure $\bar{\rho}$ on $X \times Y$. By Hopf's extension theorem, $\bar{\rho}=\rho$ on $B(X) \otimes B(Y)$; that is, $\bar{\rho}$ is the desired extension of $\rho$. //

The o-finite case is given as follows.

THEOREM 3.2.2. Let $\mu, v$ be $\sigma$-finite pre-Radon measures on topological spaces $X, Y$ respectively. Then the product measure $\rho=\mu \otimes \nu$ on $(X \times Y, B(X) \otimes B(Y))$ can be extended to a unique pre-Radon measure on $X \times Y$

Proof. Let $u$ be the paving generated by $u_{0}=\{U \times V: U \in O(X), V \in O(Y), \mu(U)<\infty, \nu(V)<\infty\}$. Assume that $W_{\alpha}, W \in U$ and $W_{\alpha} \uparrow W$. There are $U_{i} \times v_{i} \in U_{0}$ $(i=1,2, \ldots, n)$ such that $W=\bigcup_{i=1}^{n}\left(U_{i} \times V_{i}\right)$, so that we have $W \subset U_{0} \times V_{0}$, where $U_{0}=\bigcup_{i=1}^{n} U_{i}, V_{0}=\bigcup_{i=1}^{n} V_{i} \cdot$ It follows from Theorem 3.2.1 that $\mu_{U_{0}} \otimes \nu_{V_{0}}$ is extendible to a unique pre-Radon measure on $U_{0} \times V_{0}$. Thus we have

$$
\lim _{\alpha}(\mu \otimes \nu)\left(W_{\alpha}\right)=\lim _{\alpha}\left(\mu_{U_{0}} \otimes \nu_{V_{0}}\right)\left(W_{\alpha}\right)=\left(\mu_{U_{0}} \otimes \nu_{V_{0}}\right)(W)=(\mu \otimes \nu)(W) .
$$

By Theorem 2.2.2, the restriction $\left.\rho\right|_{A[U]}$ is extendible to a unique preRadon measure $\bar{\rho}$ on $X \times Y$. Since both $\rho$ and $\bar{\rho}$ are $\sigma$-finite on $B(X) \otimes B(Y), \quad \rho=\bar{\rho}$ on $B(X) \otimes B(Y)$ by Hopf's extension theorem. This completes the proof. //

Let $M^{+}(X)$ denote the set of all non-negative, extended real-valued lower semi-continuous functions on a topological space $X$.

LEMMA 3.2.3. Let $\mu$ be a semi-finite pre-Radon measure on a 
topological space $X$. If $f_{\alpha}, f \in M^{+}(X)$ and $f_{\alpha} \uparrow f$, then

$\lim _{\alpha} \int_{X} f_{\alpha} d \mu=\int_{X} f d \mu$

Proof. Suppose that $f$ is a simple function $\sum_{i=1}^{n} a_{i} \chi_{E_{i}}$, where

$E_{i} \in B(X), \quad E_{i} \cap E_{j}=\emptyset$ if $i \neq j$. The restriction $\mu_{E_{i}}$ is a pre-Radon measure by Amemiya, Okada and Okazaki [2, Theorem 5.3]. Therefore we have

$$
\left.\lim _{\alpha} \int_{E_{i}} f_{\alpha}\right|_{E_{i}} d \mu_{E_{i}}=a_{i}^{\mu}\left(E_{i}\right)
$$

by the adaption of the proof of Proposition 8.2 in Amemiya, Okada and Okazaki [2]. Then

$$
\begin{aligned}
\lim _{\alpha} \int_{X} f_{\alpha} d \mu=\lim _{\alpha} \sum_{i=1}^{n} \int_{X} f_{\alpha} x_{E_{i}} d \mu & =\left.\sum_{i=1}^{n} \underset{\alpha}{\lim } \int_{E_{i}} f_{\alpha}\right|_{E_{i}} d \mu_{E_{i}}=\sum_{i=1}^{n} a_{i} \mu\left(E_{i}\right) \\
& =\int_{X} f d \mu .
\end{aligned}
$$

Now consider an arbitrary $f \in M^{+}(X)$. If we let

$$
g_{n}=\sum_{k=1}^{n \cdot 2^{n}} \frac{k-1}{2^{n}} x_{\left\{x \in X:(k-1) / 2^{n}<f(x) \leq k / 2^{n}\right\}}+n x_{\{x \in X: f(x)>n\}},
$$

then $\lim _{n \rightarrow \infty} \int_{X} g_{n} d \mu=\int_{X} f d \mu$. Since $g_{n}$ is a simple function, it follows

from the first step that

$$
\begin{aligned}
\int_{X} f d \mu & =\lim _{n \rightarrow \infty} \int_{X} g_{n} d \mu=\lim _{n \rightarrow \infty} \lim _{\alpha} \int_{X} f_{\alpha} \wedge g_{n} d \mu \\
& =\lim _{\alpha} \lim _{n \rightarrow \infty} \int_{X} f_{\alpha} \wedge g_{n} d \mu=\lim _{\alpha} \int_{X} \lim _{n \rightarrow \infty} f_{\alpha} \wedge g_{n} d \mu \\
& =\lim _{\alpha} \int_{X} f_{\alpha} \wedge f d \mu=\lim _{\alpha} \int_{X} f_{\alpha} d \mu .
\end{aligned}
$$

LEMMA 3.2.4. Let $\mu, \nu$ be $\sigma$-finite pre-Radon measures on topological spaces $X, Y$ respectively, and let $\bar{\mu} \overline{\otimes \nu}$ denote the pre-Radon extension of $\mu \otimes \nu$ obtained in Theorem 3.2.2. Then 


$$
\overline{\mu \otimes \nu}(W)=\mu_{x} \nu_{y}\left(x_{W}(x, y)\right)
$$

for every $W \in O(X \times Y)$.

Proof. Let $u$ be the paving generated by

$$
\{U \times V: U \in O(X), V \in O(Y)\} .
$$

Given $W \in O(X \times Y)$, there exists a net $\left\{W_{\alpha}\right\} \subset U$ such that $W_{\alpha} \uparrow W$. By a way similar to Bourbaki [5, Proposition 2.6.11], the function $x \vdash \nu_{y}\left(X_{W}(x, y)\right)$ is lower semi-continuous on $X$ for all $y \in Y$. It follows from Lemma 3.2.3 that

$$
\lim _{\alpha} \nu_{y}\left(x_{W}(x, y)\right)=\nu_{y}\left(x_{W}(x, y)\right),
$$

which implies that the function $x \mapsto v_{y}\left(x_{W}(x, y)\right)$ is also lower semicontinuous for all $y \in Y$. Thus we have

$$
\overline{\mu \otimes \nu}(W)=\lim _{\alpha} \mu \otimes \nu\left(W_{\alpha}\right)=\lim _{\alpha} \mu_{x} \nu_{y}\left(x_{W_{\alpha}}(x, y)\right)=\mu_{x} \nu_{y}\left(x_{W}(x, y)\right) .
$$

Now we give Fubini's theorem.

THEOREM 3.2.5. Let $\mu, \nu$ be $\sigma$-finite pre-Radon measures on topological spaces $X, Y$ respectively, and let $\overline{\mu \otimes \nu}$ be the pre-Radon extension of $\mu \otimes \nu$ on $X \times Y$. Then, for each $B \in B(X \times Y)$,

(i) $x \mapsto \nu_{y}\left(X_{B}(x, y)\right)$ is $B(X)$-measurable for each $y \in Y$;

(ii) $y \mapsto \mu_{x}\left(X_{B}(x, y)\right)$ is $B(Y)$-measurable for each $x \in X$;

(iii) $\overline{\mu \otimes \nu}(B)=\mu_{x} \nu_{y}\left(x_{B}(x, y)\right)=\nu_{y} \mu_{x}\left(x_{B}(x, y)\right)$.

Proof. Recall that, for each $B \in B(X \times Y), X_{B}(x, y)$ is a separately Borel measurable function on $X \times Y$. Let

$$
M_{1}=\left\{E \in B(X \times Y): \nu_{y}\left(X_{E}(x, y)\right) \text { is } B(X) \text {-measurable for all } y \in Y\right\} \text {. }
$$

Since $\nu$ is $\sigma$-finite, $M_{\perp}$ is a monotone class; furthermore, we have $M_{I} \supset A[O(X \times Y)]$. Hence we have $M_{1}=B(X \times Y)$. Let

$$
M_{2}=\left\{E \in B(X \times Y): \overline{\mu \otimes \nu}(E)=\mu_{x} \nu_{y}\left(X_{E}(x, y)\right)\right\} .
$$


Since both $\mu$ and $\nu$ are $\sigma$-finite, $M_{2}$ is a monotone class. It follows from Lemma 3.2 .4 that $M_{2} \supset A[O(X \times Y)]$. Therefore $M_{2}=B(X \times Y)$. This completes the proof. //

COROLLARY 3.2.6. Let $f$ be a non-negative, extended real-valued Borel measurable function on $X \times Y$. Then

$$
\begin{aligned}
& \text { (i) } x \mapsto \int_{Y} f(x, y) d \nu(y) \text { is } B(X) \text {-measurable; } \\
& \text { (ii) } y \mapsto \int_{X} f(x, y) d \mu(x) \text { is } B(Y) \text {-measurable; } \\
& \text { (iii) } \int_{X \times Y} f(x, y) d \mu \otimes \nu=\int_{X} d \mu(x) \int_{Y} f(x, y) d \nu(y) \\
&=\int_{Y} d \nu(y) \int_{X} f(x, y) d \mu(x) .
\end{aligned}
$$

\subsection{Infinite Product of Pre-Radon Measures}

In this section, we show that the infinite product of pre-Radon measures can be extended to a unique pre-Radon measure.

At first, we give the countable product case.

THEOREM 3.3.1. Let $\mu_{n}$ be a pre-Radon probability measure on a topological space $X_{n}(n \in \mathbb{N})$. Then the product measure $\mu=\underset{n \in \mathbb{N}}{\otimes} \mu_{n}$ on $\left.\prod_{n \in \mathbb{N}} X_{n}, \underset{n \in \mathbb{N}}{\otimes} B\left(X_{n}\right)\right)$ is extendible to a unique pre-Radon probability measure on the product space $\prod_{n \in \mathbb{N}} x_{n}$.

Proof. Let $\boldsymbol{u}$ be the paving generated by $u_{0}=\left\{\prod_{n \in \mathbb{N}} U_{n}: U_{n} \in O(X)\right.$ for all $n \in \mathbb{N}$, and $U_{n}=X_{n}$ except for finitely many $\left.n \in \mathbb{N}\right\}$. 
Suppose that $W_{\alpha} \in U$ and $W_{\alpha} \uparrow \prod_{n \in \mathbb{N}} X_{n}$. Let

$$
\begin{aligned}
u_{1} & =\left\{U \in U_{0}: U \subset W_{\alpha} \text { for some } \alpha\right\} \\
& =\left\{U_{\gamma} \in U_{0}: \gamma \in \Gamma\right\}
\end{aligned}
$$

for some index set $\Gamma$. For every $\gamma \in \Gamma$, there is a $U_{n}^{\gamma} \in O\left(X_{n}\right)$ such

that $U_{\gamma}=\prod_{n \in \mathbb{N}} U_{n}^{\gamma}$. It is obvious that $\underset{\gamma \in \Gamma}{U} \prod_{n \in \mathbb{N}} U_{n}^{\gamma}=\prod_{n \in \mathbb{N}} X_{n}$. For $k \in \mathbb{N}$, let

$$
\Gamma(k)=\left\{\gamma \in \Gamma: U_{n}^{\gamma}=X_{n} \text { for all } n>k\right\}
$$

and

$$
U(k)=\underset{\gamma \in \Gamma(k)}{U} \prod_{n \in \mathbb{N}} U_{n}^{\gamma}
$$

Now we define a finitely additive set function $\nu$ on the algebra

$$
\begin{aligned}
A= & \underset{n \in \mathbb{N}}{U}\left(B\left(\prod_{i=1}^{n} x_{i}\right) \times \prod_{q>n} x_{q}\right) \text { by } \\
& \nu\left(B_{n} \times \prod_{q>n} x_{q}\right)=\overline{\bigotimes_{i=1}^{n} \mu_{i}\left(B_{n}\right)}
\end{aligned}
$$

for each $B_{n} \in B\left(\prod_{i=1}^{n} x_{i}\right)$, where $\otimes_{i=1}^{n} \mu_{i}$ denotes the pre-Radon extension of $\otimes_{i=1}^{n} \mu_{i}$ on the product space $\prod_{i=1}^{n} x_{i}$ (see Theorem 3.2.1). We claim that $v$ is well-defined. In fact, if $p_{n m}$ denotes the natural map from $\prod_{i=1}^{m} x_{i}$ to $\prod_{i=1}^{n} x_{i}$ for $m>n$, then $p_{n m}(\underbrace{m}_{i=1} \mu_{i})$ is a pre-Radon measure on $\prod_{i=1}^{n} x_{i}$ such that $p_{n m}\left(\bigoplus_{i=1}^{m} \mu_{i}\right)=\bigoplus_{i=1}^{n} \mu_{i}$ on $\left[\prod_{i=1}^{n} x_{i}, \underset{i=1}{\otimes} B\left(x_{i}\right)\right)$; hence, by Theorem 3.2.1, $p_{n m}\left[{\underset{i=1}{m} \mu_{i}}_{i=1}^{m} \mu_{i}\right.$, which means that $v$ is well-defined. 
We shall prove that $\nu$ is $\sigma$-additive. Take a decreasing sequence $\left\{E_{k}\right\}_{k \in \mathbb{N}} \subset A$, and suppose that there is an $\varepsilon>0$ for which $\nu\left(E_{k}\right\} \geq \varepsilon$ for every $k \in \mathbb{N}$. For each $E=B_{N(E)} \times \prod_{q>N(E)} X_{q} \in A$, let $E\left(x_{1}, \ldots, x_{n}\right)=\left\{\left(x_{n+1}, x_{n+2}, \ldots\right) \in \prod_{q>n} x_{q}:\right.$

$$
\left.\left(x_{1}, \ldots, x_{n}, x_{n+1}, \ldots\right) \in E\right\}
$$

and let

$$
\mu^{(n)}\left(E\left(x_{1}, \ldots, x_{n}\right)\right)=\bigoplus_{i=n+1}^{\overline{N(E)}} \mu_{i}\left(B_{N(E)}\left(x_{1}, \ldots, x_{n}\right)\right) .
$$

Moreover, if we let

$$
F_{k}=\left\{x_{1} \in X_{1}: \mu^{(1)}\left(E_{k}\left(x_{1}\right)\right) \geq \varepsilon / 2\right\},
$$

then it follows from Theorem 3.2.5 that

$$
\begin{aligned}
& \overline{N\left(E_{k}\right)} \\
& v\left(E_{k}\right)=\bigotimes_{i=1} \mu_{i}\left(E_{k}\right) \\
& =\overline{\mu_{1} \otimes\left(\begin{array}{cc}
\overline{N\left(E_{k}\right)} \\
\otimes_{i=2} & \mu_{i}
\end{array}\right)}\left(E_{k}\right) \\
& =\int_{X_{1}} \mu^{(1)}\left(E_{k}\left(x_{1}\right)\right) d \mu_{1}\left(x_{1}\right) \\
& =\int_{F_{k}} \mu^{(1)}\left(E_{k}\left(x_{1}\right)\right) d \mu_{1}\left(x_{1}\right)+\int_{F_{k}^{c}} \mu^{(1)}\left(E_{k}\left(x_{1}\right)\right) d \mu_{1}\left(x_{1}\right) \\
& \leq \mu_{1}\left(F_{k}\right)+\varepsilon / 2 .
\end{aligned}
$$

Hence $\mu_{1}\left(F_{k}\right) \geq \varepsilon / 2$, so that there exists an $\overline{x_{1}} \in X_{1}$ for which

$$
\mu^{(1)}\left(E_{k}\left(\overline{x_{1}}\right)\right) \geq \varepsilon / 2
$$

for all $k \in \mathbb{N}$. By mathematical induction, there is a sequence 


$$
\begin{aligned}
& \left.x_{n}\right]_{n \in \mathbb{N}} \in \prod_{n \in \mathbb{N}} x_{n} \text { such that } \\
& \qquad \mu^{(n)}\left(E_{k}\left(x_{1}, \ldots, \overline{x_{n}}\right) \geq \varepsilon / 2^{n}\right.
\end{aligned}
$$

for every $k \in \mathbb{N}$. Then we have $\left.x_{n}\right]_{n \in \mathbb{N}} \in \underset{k \in \mathbb{N}}{\bigcap_{k}} E_{k}$ that is, $\bigcap_{k \in \mathbb{N}} E_{k} \neq 0$. Thus $\nu$ is o-additive on $A$.

$$
\text { Since } \nu \text { is } \sigma \text {-additive, for each } \varepsilon>0 \text {, there exists a } k_{0} \in \mathbb{N}
$$

such that

$$
\left.1-\varepsilon<\nu\left(U\left(k_{0}\right)\right)=\bigotimes_{i=1}^{\overline{k_{0}} \mu_{i}} \prod_{\gamma \in \Gamma\left(k_{0}\right)} \prod_{i=1}^{k_{0}} U_{i}^{\gamma}\right) \text {. }
$$

By Theorem 3.2.1, there is a finite subset $\left\{\gamma_{1}, \ldots, \gamma_{p}\right\}$ of $\Gamma\left(k_{0}\right)$ such that

$$
\otimes_{i=1}^{k_{0}} \mu_{i}\left(\bigcup_{q=1}^{p} \prod_{i=1}^{k_{0}} U_{i}^{\gamma_{q}}\right)>1-\varepsilon
$$

Since $\left\{W_{\alpha}\right\}$ is directed, there is an $\alpha_{0}$ such that

$$
w_{\alpha_{0}} \supset{\underset{q=1}{p}}_{i=1}^{k_{0}} U_{i}^{\gamma_{q}} \times \prod_{m>k_{0}} x_{m}
$$

Thus we have $\lim _{\alpha} \mu\left(W_{\alpha}\right)=1$. By Corollary 2.2.3, $\left.\mu\right|_{A[U]}$ is extendible to a unique pre-Radon measure $\bar{\mu}$ on $\prod_{n \in \mathbb{N}} X_{n}$. Since both $\mu$ and $\bar{\mu}$ are finite, $\mu=\bar{\mu}$ on $\underset{n \in \mathbb{N}}{\otimes} B\left(X_{n}\right)$ by Hopf's extension theorem. Therefore $\bar{\mu}$ is an extension of $\mu$. This completes the proof. //

Now we prove the uncoutable case.

THEOREM 3.3.2. Let $\mu_{\lambda}$ be a pre-Radon probability measure on a topological space $X_{\lambda}(\lambda \in \Lambda)$. Then the product measure $\mu=\underset{\lambda \in \Lambda}{\otimes} \mu_{\lambda}$ on $\left(\prod_{\lambda \in \Lambda} X_{\lambda}, \underset{\lambda \in \Lambda}{\otimes} B\left(X_{\lambda}\right)\right)$ is extendible to a unique pre-Radon measure on the 
product space $\prod_{\lambda \in \Lambda} X_{\lambda}$.

Proof. Let $u$ be the paving generated by $u_{0}=\left\{\prod_{\lambda \in \Lambda} U_{\lambda}: U_{\lambda} \in O\left(X_{\lambda}\right)\right.$ for every $\lambda \in \Lambda$ and

$$
\left.U_{\lambda}=X_{\lambda} \text { except for finitely many } \lambda\right\}
$$

Take a net $\left\{W_{\alpha}\right\} \subset U$ such that $W_{\alpha} \uparrow \prod_{\lambda \in \Lambda} X_{\lambda}$, and let $c=\sup _{\alpha} \mu\left(W_{\alpha}\right)$. Then there is a sequence $\left\{\alpha_{n}\right\}_{n \in \mathbb{N}}$ such that $c=\lim _{n \rightarrow \infty} \mu\left(W_{\alpha_{n}}\right)=\mu\left(\underset{n \in \mathbb{N}}{U} W_{\alpha_{n}}\right)$. For simplicity, let $W=\underset{n \in \mathbb{N}}{U} W_{\alpha_{n}}$. For each $\alpha$, we can write $w_{\alpha}=\bigcup_{i=1}^{N(\alpha)} \prod_{\lambda \in \Lambda} U_{\lambda}^{\alpha, i}$, where $\prod_{\lambda \in \Lambda} U_{\lambda}^{\alpha, i} \in u_{0}(i=1,2, \ldots, N(\alpha))$. If we let

$\Lambda_{0}=\left\{\lambda \in \Lambda: U_{\lambda}^{\alpha_{n}, i}=X_{\lambda}\right.$ for every $n \in \mathbb{N}$ and every $\left.i=1,2, \ldots, N\left(\alpha_{n}\right)\right\}$, then $\Lambda_{1}=\Lambda-\Lambda_{0}$ is a countable set. Since $W=q_{I}(W) \times \prod_{\lambda \in \Lambda_{0}} X_{\lambda}$, we have $\mu(W)=\left(\underset{\lambda \in \Lambda_{1}}{\otimes} \mu_{\lambda}\right)\left(q_{\perp}(W)\right)$, where $q_{I}$ is the projection of $\prod_{\lambda \in \Lambda} x_{\lambda}$ to $\prod_{\lambda \in \Lambda_{1}} x_{\lambda}$ At first, assume that $S\left(\mu_{\lambda}\right)=X_{\lambda}$ for all $\lambda \in \Lambda$. Suppose that $c<1$; then there is an $\alpha_{0}$ such that $\left(\underset{\lambda \in \Lambda_{I}}{\otimes} \mu_{\lambda}\right)\left(q_{I}\left(W_{\alpha_{0}}\right)-q_{I}(W)\right)>0$ since $\lim _{\alpha}\left(\underset{\lambda \in \Lambda_{\perp}}{\otimes} \mu_{\lambda}\right)\left(q_{\perp}\left(W_{\alpha}\right)\right)=1$ by Theorem 3.3.1. Thus there exists an $i$ $\left(1 \leq i \leq N\left(\alpha_{0}\right)\right)$ such that $\left(\otimes_{\lambda \in \Lambda_{\perp}}^{\otimes} \mu_{\lambda}\right)\left(\prod_{\lambda \in \Lambda_{\perp}} U_{\lambda}^{\alpha_{0}, i}-q_{\perp}(W)\right)>0$. Since 


$$
\begin{aligned}
& \left(\underset{\lambda \in \Lambda_{0}}{\otimes} \mu_{\lambda}\right)\left(\prod_{\lambda \in \Lambda_{0}} U_{\lambda}^{\alpha_{0}, i}\right)>0 \text {, we have } \\
& c=\mu\left(W \cup W_{\alpha_{0}}\right) \geq \mu\left(w \cup \prod_{\lambda \in \Lambda} U_{\lambda}^{\alpha_{0}, i}\right)
\end{aligned}
$$

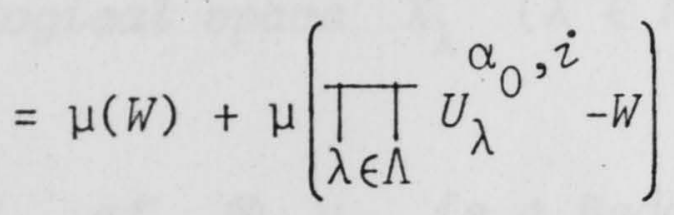

$$
\begin{aligned}
& =\mu(W)+\mu\left(\left(\prod_{\lambda \in \Lambda_{1}} U_{\lambda}^{\alpha_{0}, i}-q_{1}(W)\right) \times \prod_{\lambda \in \Lambda_{0}} U_{\lambda}^{\alpha_{0}, i}\right) \\
& =\mu(W)+\left(\underset{\lambda \in \Lambda_{\perp}}{\otimes} \mu_{\lambda}\right)\left(\prod_{\lambda \in \Lambda_{\perp}} U_{\lambda}^{\alpha_{0}, i}-q_{\perp}(W)\right) \cdot\left(\underset{\lambda \in \Lambda_{0}}{\otimes} \mu_{\lambda}\right)\left(\prod_{\lambda \in \Lambda_{0}} U_{\lambda}^{\alpha_{0}, i}\right)>c,
\end{aligned}
$$

which is a contradiction. Thus $c=1$.

Now we consider the general case. Let $\nu=\left.\mu_{\lambda}\right|_{Y}$, where

$$
\begin{aligned}
& y_{\lambda}=S\left(\mu_{\lambda}\right) \neq \not \text {. Since } W_{\alpha} \cap \prod_{\lambda \in \Lambda} Y_{\lambda} \uparrow \prod_{\lambda \in \Lambda} Y_{\lambda} \text {, it follows that } \\
& \lim _{\alpha}\left(\otimes_{\lambda \in \Lambda} \mu_{\lambda}\right)\left(W_{\alpha}\right)=\lim _{\alpha}\left(\underset{\lambda \in \Lambda}{\otimes} \nu_{\lambda}\right)\left(W_{\alpha} \cap \prod_{\lambda \in \Lambda} y_{\lambda}\right) \\
& =\left(\underset{\lambda \in \Lambda}{\otimes} \nu_{\lambda}\right)\left(\prod_{\lambda \in \Lambda} Y_{\lambda}\right)=\left(\underset{\lambda \in \Lambda}{\otimes} \mu_{\lambda}\right)\left(\prod_{\lambda \in \Lambda} X_{\lambda}\right) .
\end{aligned}
$$

It follows from Corollary 2.2 .3 that $\left.\mu\right|_{A[U]}$ can be extended to a unique pre-Radon measure $\bar{\mu}$ on the product space $\prod_{\lambda \in \Lambda} X_{\lambda}$. Since both $\mu$ and $\bar{\mu}$ are finite, $\mu=\bar{\mu}$ on $\underset{\lambda \in \Lambda}{\otimes} B\left(X_{\lambda}\right)$ by Hopf's extension theorem. This completes the proof. //

Finally we consider when the pre-Radon extension $\bar{\otimes} \overline{\lambda \in \Lambda}_{\lambda}$ of $\underset{\lambda \in \Lambda}{\otimes} \mu_{\lambda}$ is a Radon measure. The following lemma is well-known (see, for example, Bourbaki [5, Théorème 4.2]).

LEMMA 3.3.3. Let $\mu_{n}$ be a Radon probability measure on a topological 
space $X_{n}(n \in N)$. Then the pre-Radon extension $\bar{\otimes} \mu_{n \in \mathbb{N}}$ on $\prod_{n \in \mathbb{N}} X_{n}$ of $\otimes \mu_{n}$ is a Radon measure.

THEOREM 3.3.4. Let $\mu_{\lambda}$ be a Radon probability measure on a topological space $X_{\lambda}(\lambda \in \Lambda)$. Then the pre-Radon extension $\overline{\lambda \in \Lambda}_{\lambda}$ on $\prod_{\lambda \in \Lambda} X_{\lambda}$ of $\underset{\lambda \in \Lambda}{\otimes} \mu_{\lambda}$ is a Radon measure if and only if $S\left(\mu_{\lambda}\right) \in K\left(X_{\lambda}\right)$ except countably many $\lambda \in \Lambda$.

Proof. It suffices to prove the 'only if' part since the 'if' part is derived from Lemma 3.3.3. Suppose that $\overline{\otimes \in \Lambda} \mu_{\lambda}$ is a Radon measure. Let us assume that there exists an uncountable subset $\Lambda_{0}$ of $\Lambda$ such that $S\left(\mu_{\lambda}\right)$ is non-compact in $X_{\lambda}$ for all $\lambda \in \Lambda_{0}$. Without loss of generality, we may assume that $\Lambda_{0}=\Lambda$. We shall show that $\overline{\otimes \in \Lambda} \mu_{\lambda}(K)=0$ for all $K \in K\left(\prod_{\lambda \in \Lambda} x_{\lambda}\right)$. Given $K \in K\left(\prod_{\lambda \in \Lambda} x_{\lambda}\right)$, let

$$
\Lambda_{n}=\left\{\lambda \in \Lambda: \mu_{\lambda}\left(p_{\lambda}(K)\right)<1-1 / n\right\},
$$

then $\Lambda=\bigcup_{n \in \mathbb{N}} \Lambda_{n}$, where $p_{\lambda}$ is the projection of $\prod_{\lambda \in \Lambda} X_{\lambda}$ to $X_{\lambda}$. Since $\Lambda$ is uncoutable, there is an $n \in \mathbb{N}$ such that $\Lambda_{n}$ is an infinite set; hence $\Lambda_{n}$ includes an infinite sequence $\left\{\lambda_{i}\right\}_{i \in \mathbb{N}}$. Then we have 


$$
\begin{aligned}
& {\underset{\lambda \in \Lambda}{\otimes} \mu_{\lambda}}_{\lambda}(K) \leq \varlimsup_{\lambda \in \Lambda}^{\otimes} \mu_{\lambda}\left(\prod_{\lambda \in \Lambda} p_{\lambda}(K)\right) \\
& \leq \bigotimes_{\lambda \in \Lambda}^{\otimes} \mu_{\lambda}\left(\prod_{i \in \mathbb{N}} p_{\lambda}(K) \times \prod_{\lambda \neq \lambda_{i}} X_{\lambda}\right) \\
& =\lim _{k \rightarrow \infty}\left(\bigotimes_{\lambda \in \Lambda}^{\otimes} \mu_{\lambda}\right)\left(\prod_{i=1}^{k} p_{\lambda_{i}}(K) \times \prod_{\lambda \neq \lambda_{i}} x_{\lambda}\right) \\
& =\lim _{k \rightarrow \infty} \prod_{i=1}^{k} \mu_{\lambda_{i}}\left(p_{\lambda_{i}}(K)\right) \\
& \leq \lim _{k \rightarrow \infty}(1-1 / n)^{k}=0 \text {. }
\end{aligned}
$$

Thus

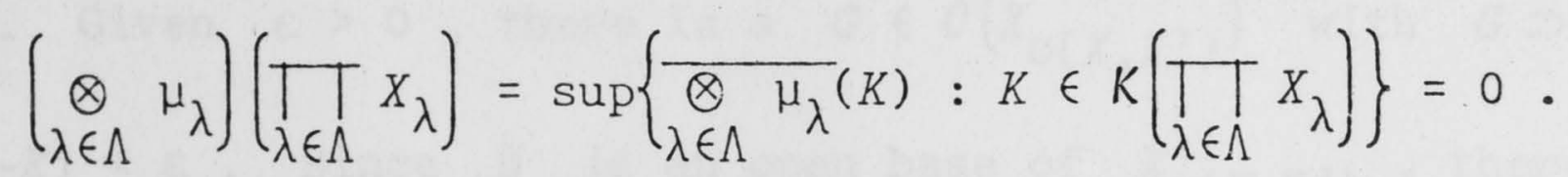

This is a contradiction. //

As an application, we have a pre-Radon measure which is not a Radon measure.

EXAMPLE 3.3.5. Let $\Lambda$ be an uncountable index set, and let $d \mu_{\lambda}(x)=(1 / \sqrt{2 \pi}) \exp \left(-x^{2} / 2\right)$ on $\mathbb{R}$ for $\lambda \in \Lambda$, where $d x$ is the Lebesgue measure. Then the pre-Radon extension $\bigotimes_{\lambda \in \Lambda}^{\otimes} \mu_{\lambda}$ on $\mathbb{R}^{\Lambda}$ is not a Radon measure by Theorem 3.3.4.

\subsection{Cylindrical Measures}

The pre-Radon extension theorems can be applied to extend some cylindrical measures.

THEOREM 3.4.1. Let $X$ be a locally convex space and $U$ be a paving generated by a $\sigma\left(X, X^{\prime}\right)$-open base included in $Z\left(X, X^{\prime}\right)$. A finite realvalued, finitely additive set function $\mu$ on $A[U]$ is extendible to a 
unique Radon measure on $(X, \rho)$ for a topology $\rho$ (not necessarily a Zocally convex topology) stronger then $\sigma\left(X, X^{\prime}\right)$ if and only if $\mu$ satisfies the following conditions:

(i) $\mu(V)=\sup \left\{\mu(F): U \supset F \in A[U] \cap F\left(X_{\sigma\left(X, X^{\prime}\right)}\right)\right\}$ for each

$$
U \in U \text {; }
$$

(ii) $\mu(X)=\sup \left\{\mu^{0}(K): K \in K((X, \rho))\right\}$, where

$$
\mu^{0}(K)=\inf \{\mu(U): K \subset U \in U\} \text {. }
$$

Proof. By Corollary 2.2.4, $\mu$ can be extended to a unique Radon measure $\bar{\mu}$ on $X_{\sigma\left(X, X^{\prime}\right)}$. We shall show that $\bar{\mu}(K)=\mu^{0}(K)$ for all $K \in X(X)$. Given $\varepsilon>0$, there is a $G \in O\left(X_{\sigma\left(X, X^{\prime}\right)}\right)$ with $G \supset K$ such that $\bar{\mu}(G-K)<\varepsilon$. Since $U$ is an open base of $X_{\sigma\left(X, X^{\prime}\right)}$, there is a $U \in U$ such that $K \subset U \subset G$, which means that $0 \leq \mu(U)-\bar{\mu}(K)<\varepsilon$. Thus we have $\mu^{0}(K)=\bar{\mu}(K)$ for all $K \in K(X)$. Then there is a $\sigma$-compact subset $L$ of $(X, \rho)$ such that $\bar{\mu}(L)=\bar{\mu}(X)$, so that the identity map $\imath$ from $X_{\sigma\left(X, X^{\prime}\right)}$ onto $(X, \rho)$ is $\bar{\mu}$-Lusin-measurable (for the definition of Lusin-measurability see, for example, Schwartz [43, Definition 1.9]). Therefore the image measure $\iota(\bar{\mu})$ is a Radon measure, which satisfies $\iota(\bar{\mu})(A)=\mu(A)$ for every $A \in A[U]$. The uniqueness of this extension is obvious. $\quad / /$

The following corollary is a generalization of Theorem 1 of Dudley, Feldman and LeCam [7], which is a variant of results of Prokhorov [38].

COROLLARY 3.4.2. Let $X$ be a locally convex space and $\mu$ a cylinder set measure on $X$. Then $\mu$ is extendible to a unique Radon measure on $(X, \rho)$ for a topology $\rho$ stronger than $\sigma\left(X, X^{\prime}\right)$ if and only if

$$
\mu(X)=\sup \left\{\mu^{0}(K): K \in K((X, \rho))\right\},
$$

where

$$
\mu^{0}(K)=\inf \left\{\mu(U): K \subset U \in Z\left(X, X^{\prime}\right) \cap O\left(X X_{\sigma\left(X, X^{\prime}\right)}\right)\right\} .
$$


The following two theorems are shown by Schachermayer and Tortrat respectively. Here we prove them by using the pre-Radon extension theorems.

THEOREM 3.4.3 (Schachermayer [41, Lemma 4]). Let $\mu$ be a cylinder set measure on a locally convex space $X$. Then $\mu$ is extendible to a unique pre-Radon measure on $X_{\sigma\left(X, X^{\prime}\right)}$ if and only if $\sup _{\alpha} \mu\left(U_{\alpha}\right)=\mu(X)$ for each net $\left\{U_{\alpha}\right\} \subset Z\left(X, X^{\prime}\right) \cap O\left(X_{\sigma\left(X, X^{\prime}\right)}\right)$ such that $U_{\alpha} \uparrow X$.

Proof. It is obvious that

$$
u=Z\left(X, X^{\prime}\right) \cap O\left(X_{\sigma\left(X, X^{\prime}\right)}\right)=\underset{F \in F D\left(X^{\prime}\right)}{U} \pi_{F}^{-1}\left(O\left(X / F^{\prime}\right)\right)
$$

is a paving. The set function $\left.\mu\right|_{A[U]}$ can be extended to a unique pre-Radon measure $\bar{\mu}$ on $X_{\sigma\left(X, X^{\prime}\right)}$ by Corollary 2.2.3. Since $\pi_{F}(\bar{\mu})$ is a Radon measure on $X / F^{1}, \bar{\mu}=\mu$ on $\pi_{F}^{-1}\left(B\left(X / F^{1}\right)\right)$, which implies that $\bar{\mu}$ is an extension of $\mu$. Suppose that there is another pre-Radon extension $\nu$ of $\mu$. Then $\nu=\bar{\mu}$ since $U$ is an open base of $X_{\sigma\left(X, X^{\prime}\right)}$. //

THEOREM 3.4.4 (Tortrat [46, Theorem 7]). Let $X$ be a Zocally convex space and $\mu$ a pre-Radon measure on $X_{\sigma\left(X, X^{\prime}\right)}$. Then $\mu$ is extendible to a unique pre-Radon measure on $X$ if and only if $\sup _{\alpha} \mu\left(U_{\alpha}\right)=\mu(U)$ for each net $\left\{U_{\alpha}\right\} \subset O(X) \cap B\left(X_{\sigma}\right)$ such that $U_{\alpha} \uparrow U \in B\left(X_{\sigma}\right)$.

Proof. It suffices if we prove the 'if' part. Let $u$ be the paving generated by

$$
\left\{x+U_{p}: x \in X, p \text { is a continuous seminorm on } X\right\},
$$

where $U_{p}=\{x \in X: p(x)<1\}$; then $u \subset O(X) \cap B\left(X_{\sigma}\right)$. For each $U \in U$, there are $x_{i j} \in X$ and continuous seminorms $p_{i j}(i=1,2, \ldots, n$; $j=1,2, \ldots, m)$ such that $U=\bigcap_{i=1}^{n} \bigcup_{j=1}^{m}\left(x_{i j}+p_{i j}\right)$. Since

$$
x_{i j}+U_{p_{i j}}=\underset{k \in \mathbb{N}}{U}\left(x_{i j}+\left\{y \in X: p_{i j}(x) \leq 1-1 / k\right\}\right),
$$


given $\varepsilon>0$ there is a $k_{i j} \in \mathbb{N}$ such that

$$
\mu\left(x+\left\{y \in X: p_{i j}(y) \leq 1-1 / k_{i j}\right\}\right) \geq \mu\left(x_{i j}+u_{i j}\right)-\varepsilon / 2^{i+j} .
$$

Let

$$
F_{i j}=x_{i j}+\left\{y \in X: p_{i j}(y) \leq 1-1 / k_{i j}\right\}
$$

then

$$
F=\bigcap_{i=1}^{n} \bigcup_{j=1}^{m} F_{i j} \in A[U] \cap F(X)
$$

Thus we have

$$
\begin{aligned}
\mu(U-F) & =\mu\left(\bigcap_{i=1}^{n} \bigcup_{j=1}^{m}\left(x_{i j}+P_{p_{i j}}\right)-\bigcap_{i=1}^{n} \bigcup_{j=1}^{m} F_{i j}\right) \\
& \left.\leq \mu(\bigcup_{i=1}^{n}\left(\bigcup_{j=1}^{m}\left(x_{i j}+U_{p_{i j}}\right)\right)-\underbrace{m}_{j=1} F_{i j})\right) \\
& \leq \mu\left(\bigcup_{i=1}^{n} \bigcup_{j=1}^{m}\left(\left(x_{i j}+p_{p_{i j}}\right)-F_{i j}\right)\right) \\
& \leq \sum_{i=1}^{n} \sum_{j=1}^{m} \varepsilon / 2^{i+j}<\varepsilon .
\end{aligned}
$$

By Theorem 2.2.1, $\left.\mu\right|_{A[u]}$ is extendible to a unique pre-Radon measure $\bar{\mu}$. on $X$. Since $\bar{\mu}=\mu$ on $u$, we have $\bar{\mu}=\mu$ on $O\left(X_{\sigma\left(X, X^{\prime}\right)}\right)$. Note that $\left.\bar{\mu}\right|_{B\left(X_{\sigma\left(X, X^{\prime}\right)}\right)}$ is a pre-Radon measure on $X_{\sigma\left(X, X^{\prime}\right)}$, for $\left.\bar{\mu}\right|_{B\left(X_{\sigma\left(X, X^{\prime}\right)}\right)}$ is $\tau$-smooth on $X_{\sigma\left(X, X^{\prime}\right)}$ and $X_{\sigma\left(X, X^{\prime}\right)}$ is a completely regular space. Thus $\bar{\mu}=\mu$ on $B\left(X_{\sigma\left(X, X^{\prime}\right)}\right)$; that is, $\bar{\mu}$ is an extension of $\mu$. The uniqueness follows from the fact that $U$ is an open base of $X$.

In the following example, we show that all cylindrical measures on $\left(X, C\left(X, X^{\prime}\right)\right)$ which can be extended to the Borel field $B(X)$ have not necessarily unique extensions.

EXAMPLE 3.4.5. Let $\mu$ be the Dieudonné measure on $[0, \Omega]$ in 
Example 1.2.15. Then the function $\sigma$ from $[0, \Omega]$ into $\mathbb{R}^{C([0, \Omega])}$ defined by $\left.\sigma(x)=(f(x))_{f \in C(}[0, \Omega]\right)$ for $x \in[0, \Omega]$ is a homeomorphism by Gillman and Jerison [11, Theorem 11.3]. We claim that $\sigma(\mu)$ is not regular. In fact, if so, then $\left.\sigma(\mu)\right|_{\sigma([0, \Omega])}$ is regular, which is a contradiction. Let $\mu_{0}=\left.\mu\right|_{B *([0, \Omega])}$; then there is a Radon measure $\nu$ on $[0, \Omega]$ such that $\int_{[0, \Omega]} f d \nu=\int_{[0, \Omega]} f d \mu_{0}$ for all $f \in C([0, \Omega]$ ) (see, for example, Hewitt and Ross $[18, \S 11])$. Thus $\nu=\mu_{0}$ on $B^{*}([0, \Omega])$ by Varadarayan $[47$, Theorem [.5]), so that $\sigma(\nu)=\sigma(\mu)$ on

$$
B^{*}\left(\mathbb{R}^{C([0, \Omega])}\right)=C\left(\mathbb{R}^{C([0, \Omega])},\left(\mathbb{R}^{C([0, \Omega])}\right)\right) .
$$

Therefore the cylindrical measure $\left.\sigma(\mu)\right|_{C\left(\mathbb{R}^{C([0, \Omega])},\left(\mathbb{R}^{C([0, \Omega])^{\prime}}\right)^{\prime}\right)}$ has two Borel extensions $\sigma(\mu)$ and $\sigma(\nu)$, though $\sigma(\mu) \neq \sigma(\nu)$ since $\sigma(\mu)$ is not regular and $\sigma(\mu)$ is regular.

Schacharmayer has given a cylindrical measure which can not be extended to a pre-Radon measure.

EXAMPLE 3.4.6 (Schachermayer $[42$, Beispiel 7.5]). Let $X=C([0, \Omega])$; then $x_{\{\Omega\}} \in X^{\prime \prime}$. If we let $\tilde{\mu}=\left.\delta_{\chi_{\{\Omega\}}}\right|_{C\left(X^{\prime \prime}, X^{\prime}\right)}$, then $\tilde{\mu}$ is a $\sigma$-additive measure on $\left(X^{\prime \prime}, C\left(X^{\prime \prime}, X^{\prime}\right)\right)$. Since $X$ is a $\tilde{\mu}$-thick subset of $X^{\prime \prime}$, $\mu=\tilde{\mu}$ is also $\sigma$-additive on $C\left(X, X^{\prime}\right)=X \cap C\left(X^{\prime \prime}, X^{\prime}\right)$; that is, $\mu$ is a cylindrical measure on $X$. However, $\mu$ can not be extendible to a preRadon measure on $X_{\sigma\left(X, X^{\prime}\right)}$. 


\section{CHAPTER IV}

\section{MEASURE-COMPACTNESS AND BOREL MEASURE-COMPACTNESS}

\subsection{Introduction and Preliminary Results}

Since Moran introduced measure-compact spaces and strongly measurecompact spaces in [30] and [31], many mathematicians have worked on their properties. On the other hand, Gardner [10] has given a similar notion for Borel measures, that is, Borel measure-compact spaces, $B^{*}$-spaces, $H B^{*}$ spaces; and independently Adamski [1] has defined $B^{*}$-spaces, $H B^{*}$-spaces, where a $B^{*}$-space is called a weak $\tau$-space and an $H B^{*}$-space is called a $\tau$-space. In this section, we give some results which introduce two new classes of topological spaces, weak Radon spaces and Borel regular spaces. We also illustrate the relationships between these spaces (see the diagram on page 47).

In $\$ 4.2$, we study whether those spaces are closed under countable unions.

In $\$ 4.3$, we examine the hereditariness of those spaces.

All topological spaces in this chapter are supposed to be completely regular, and all measures considered are finite.

A topological space $X$ is said to be measure-compact (respectively strongly measure-compact) if every Baire measure on $X$ is $\tau$-smooth (respectively tight). The following results are proved by Moran:

(I) ([30, Theorem 2.1]). A topological space $X$ is measure-compact if and only if every non-zero Baire measure on $X$ has a non-empty support.

(II) ([31, Proposition 4.4]). A topological space $X$ is strongly measure-compact if and only if, for every Baire measure $\mu$ on $X$, there exists a $K \in K(X)$ such that $\mu^{*}(K)>0$.

We call a topological space $X$ Borel measure-compact if every regular 
Borel measure is $\tau$-smooth. A topological space $X$ is a $B^{*}$-space if each Borel measure on $X$ is weakly $\tau$-smooth, and $X$ is an $H B^{*}$-space if each subspace of $X$ is a $B^{*}$-space. Gardner has given the following:

(III) ([10, Theorem 3.1]). A topological space $X$ is Borel measurecompact if and only if every non-zero regular Borel measure on $X$ has a non-empty support.

(IV) ([10, Theorem 4.1]). A topological space $X$ is a $B^{*}$-space if and only if every non-zero Borel measure on $X$ has a non-empty support.

(V) ([10, Theorem 5.2]). A topological space $X$ is an $H B^{*}$-space if and only if every Borel measure on $X$ is $\tau$-smooth.

A topological space $X$ is called a Borel-regular space if every Borel measure is regular; Yamasaki has introduced this concept. We call a topological space $X$ a weak Radon space (respectively a Radon space) if every regular Borel measure (respectively Borel measure) is a Radon measure. It is well-known that all standard spaces are Radon spaces. Recall that a topological space $X$ is a standard space if there is a continuous surjection from a Polish space to $X$.

For the spaces defined above, we have the following relationships:

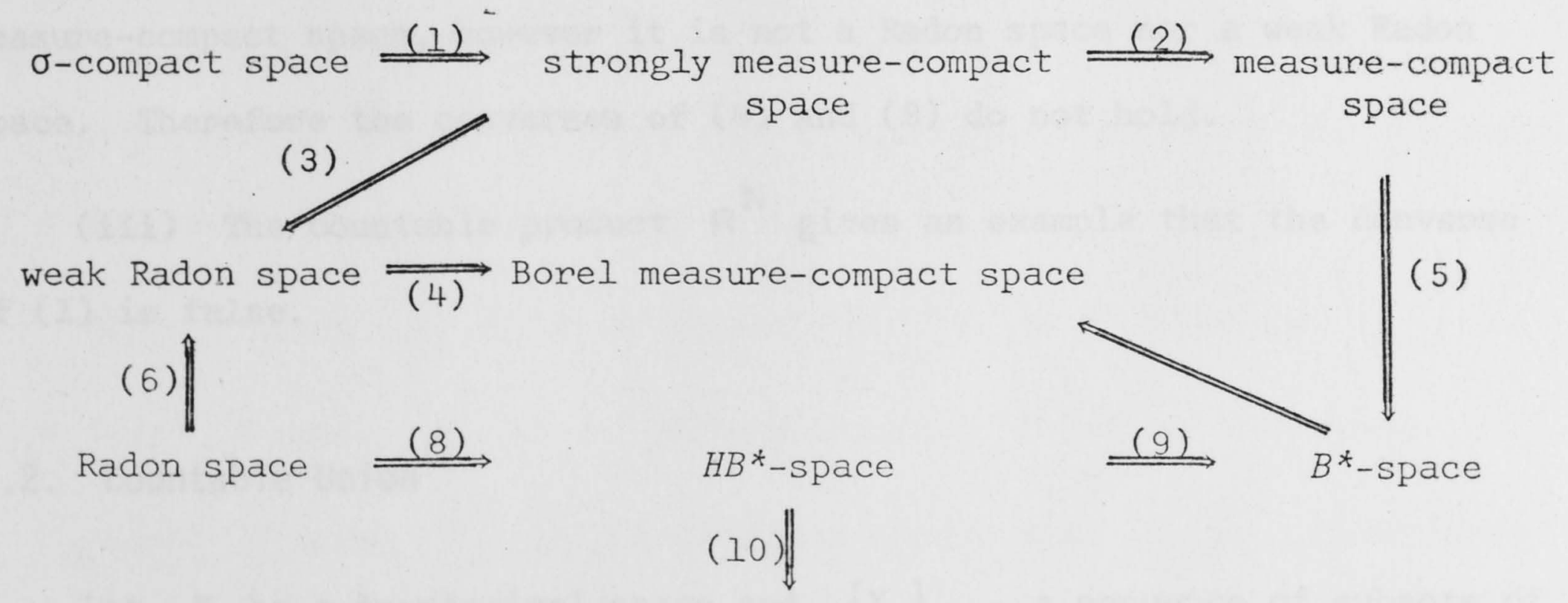

Borel-regular space

$(1),(2),(4),(6),(8)$ and (9) are obvious from the definitions. (7) is evident from (III) and (IV). (I0) is implied by Gardner [10, Theorem 6.1]. We show (3) and (5). 
THEOREM 4.1.1. (i) A strongly measure-compact space is a weak Radon space.

(ii) A measure-compact space is a $B^{*}$-space.

Proof. (i) Let $\mu$ be a regular Borel measure on a strongly measurecompact space $X$. Then $\nu=\left.\mu\right|_{B^{*}(X)}$ is a tight measure. Theorem 2.1.3 implies that $\nu$ is extendible to a Radon measure $\bar{\nu}$ on $X$. Then $\mu=\bar{v}$ by Kirk [22, Corollary 1.15].

(ii) Let $\mu$ be a non-zero Borel measure on a measure-compact space $X$. Then $S^{*}\left(\left.\mu\right|_{B^{*}(X)}\right) \neq \not D$. By Proposition 1.2 .12 and (IV), $X$ is a $B^{*}$-space. $\quad / /$

EXAMPLE 4.1.2. (i) The Dieudonne measure in Example 1.2.15 shows that the converses of (6) and (9) do not hold, and that a $B^{*}$-space is not necessarily a Borel-regular space.

(ii) Let $A$ be a non-Lebesgue measurable subset of $[0,1]$ such that $\mu^{*}(A)>0$ and $\mu_{*}(A)=0$, where $\mu$ is the Lebesgue measure on $[0,1]$. Then the restriction $\mu_{A}$ is a regular $\tau$-smooth Borel measure on $A$, but it is not a Radon measure. This $A$ is an $H B^{*}$-space, hence a Borel measure-compact space, however it is not a Radon space nor a weak Radon space. Therefore the converses of (4) and (8) do not hold.

(iii) The countable product $\mathbb{R}^{\mathbb{N}}$ gives an example that the converse of (1) is false.

\subsection{Countable Union}

Let $X$ be a topological space and $\left\{X_{n}\right\}_{n \in N}$ a sequence of subsets of $X$ such that $X=\underset{n \in \mathbb{N}}{U} X_{n}$. Gardner [10, Theorem 3.7, Theorem 7.4] has proved that if every $X_{n}$ is Borel measure-compact (respectively $C^{*}-$ embedded and measure-compact), then $X$ is Borel measure-compact (respectively 
measure-compact). In this section, we show that some spaces defined in $\$ 4.1$ have the same property.

PROPOSITION 4.2.1. Let $X$ be a topological space and $\left\{X_{n}\right\}_{n \in \mathbb{N}} a$ sequence of subsets of $X$ such that $X=\bigcup_{n \in \mathbb{N}} X_{n}$.

(i) If every $X_{n}$ is Baire-embedded and measure-compact, then $X$ is measure-compact.

(ii) If every $X_{n}$ is Baire-embedded and strongly measure-compact,

then $X$ is strongly measure-compact.

Proof. (i) We can prove it by the way similar to Gardner [10, Theorem 3.7].

(ii) Let $\mu$ be a Baire measure on $X$; then there exists an $n \in \mathbb{N}$ such that $\mu^{*}\left(X_{n}\right)>0$. Since $B^{*}\left(X_{n}\right)=X_{n} \cap B^{*}(X)$, the restriction $\mu_{X}$ is a non-zero Baire measure on $X_{n}$. By (II) in $\$ 4.1$, there is a $K \in K(X)$ such that $\left(\mu_{X}\right) *\left(K_{n}\right)>0$. For any $B \in 3^{*}(X)$ with $B \supset K$, we have

$$
\mu(B) \geq \mu_{X}\left(B \cap K_{n}\right) \geq\left(\mu_{X}\right) *(K)>0
$$

which means that $\mu^{*}(K)>0$. Hence $X$ is strongly measure-compact.

PROPOSITION 4.2.2. Suppose that a topological space $X$ is the union of a sequence $\left\{X_{n}\right\}_{n \in \mathbb{N}}$ of $i$ ts subsets.

(i) If every $X_{n}$ is a $B^{*}$-space, then $X$ is a $B^{*}$-space.

(ii) If every $X_{n}$ is an $H B^{*}$-space, then $X$ is an $H B^{*}$-space.

(iii) If every $X_{n}$ is a weak Radon space, then $X$ is a weak Radon space.

(iv) If every $X_{n}$ is a Radon space, then $X$ is a Radon space.

Proof. Let $\mu$ be an arbitrary Borel measure on $X$. 
(i) There is an $n \in \mathbb{N}$ such that $\mu^{*}\left(X_{n}\right)>0$. Then the restriction $\mu_{X_{n}}$ is a non-zero Borel measure on $X_{n}$. From the assumption, $S\left(\mu_{X_{n}}\right) \neq \varnothing ;$ that is, there is an $x \in S\left(\mu_{X_{n}}\right)$. For each $U \in V^{\prime}(x)$, $\mu(U) \geq \mu_{X_{n}}\left(U \cap X_{n}\right)>0$. Thus $x \in S(\mu)$. By (IV) in $\$ 4.1, X$ is a $B^{*}$-space.

(i) Take any subset $A$ of $X$. Since $X_{n}$ is an $H B$-space, $A \cap X_{n}$ is a $B^{*}$-space. By $(i), A=\underset{n \in \mathbb{N}}{U}\left(A \cap X_{n}\right)$ is a $B^{*}$-space.

(iii) Suppose that $\mu$ is regular on $X$; then $\mu_{X}$ is regular on $X_{n}$ for every $n \in N$. Given $\varepsilon>0$, there is a $K_{n} \in K\left(X_{n}\right)$ such that $\mu_{X}\left(X_{n}-K_{n}\right)<\varepsilon / 2^{n}$ since $\mu_{X}$ is a Radon measure. Then we have

$$
\mu\left(X-\bigcup_{n \in \mathbb{N}} K_{n}\right) \leq \sum_{n \in \mathbb{N}} \mu_{X}\left(X_{n}-K_{n}\right)<\varepsilon,
$$

which implies that $\mu(X)=\sup \{\mu(K): K \in K(X)\}$. It follows fhom the regularity of $\mu$ that $\mu$ is a Radon measure.

(iv) For any $B \in B(X), B \cap X_{n} \in B\left(X_{n}\right)$. By the argument in (iii), we have

$$
\mu(B)=\sup \{\mu(K): K \in K(X)\} ; \quad K \subset B
$$

that is, $\mu$ is a Radon measure. //.

\subsection{Hereditariness}

Every Baire set of a topological space $X$ is measure-compact by Moran [31, Proposition 5.1], every closed subset is also measure-compact by Kirk [21, Theorem 3.5]. Here we have the following:

PROPOSITION 4.3.1. (i) Let $X$ be a measure-compact space and $A$ a 
subset of $X$. Suppose that, for each $F \in F(X)$ with $F \cap A=\varnothing$, there exists $a \quad B \in B(X)$ such that $B \supset A$ and $B \cap F=\not D$. Then $A$ is measurecompact.

(ii) Every $F_{\sigma}$-subset of a normal, measure-compact space is measurecompact.

Proof. (i) We can prove it by the same way as in the proof of Moran [3, Proposition 5.1].

(ii) Since a closed subset of a normal space is Baire-embedded, every $F_{\sigma}$-subset is measure-compact by Proposition 4.2.1. $/ /$.

If $X$ is a strongly measure-compact space, then all Baire subsets of $X$ are also strongly measure-compact by Moran [31, Proposition 4.5], and all closed subsets of $X$ are also strongly measure-compact by Mosiman and Wheeler [33, Proposition 3.2]. In particular, if $X$ is normal and strongly measure-compact, then each $F_{\sigma}$-subset is strongly measure-compact by Proposition 4.2.1.

Now we consider subspaces of a $B^{*}$-space. Every $F_{\sigma}$-subset of a $B^{*}$-space is a $B^{*}$-space by Adamski [1, Theorem 3.7]. We have

THEOREM 4.3.2. Let $X$ be a $B^{*}$-space. Suppose a subset $A$ of $X$ satisfies the condition that for every $F \in F(X)$ with $F \cap A=\varnothing$, there is a $B \in B^{*}(X)$ for which $B \supset A$ and $B \cap F=\varnothing$. Then $A$ is a $B^{*}$-space. In particular, every Baire set is a $B^{*}$-space.

Proof. If $\mu$ is a Borel measure on $A$, then $\iota(\mu)$ is weakly $\tau$-smooth, where $\iota$ is the natural injection from $A$ to $X$. Then, by Gardner [10, Lemma 4.2],

$$
\mu(A)=\iota(\mu)(X)=\inf \{\iota(\mu)(G): S(\iota(\mu)) \subset G \in O(X)\} .
$$

Suppose that $S(\iota(\mu)) \cap A=\varnothing$; then there is a $B \in B(X)$ such that $B \supset A$ and $B \cap S(\iota(\mu))=\varnothing$. It follows that 


$$
\begin{aligned}
0=\mu\left(B^{c}\right) & =\inf \left\{c(\mu)(U): B^{c} \subset U \in U(X)\right\} \\
& \geq \inf \{\iota(\mu)(G): S(\iota(\mu)) \subset G \in O(X)\} \\
& =\mu(A)>0,
\end{aligned}
$$

which is a contradiction. Then there is an $x \in S(\iota(\mu)) \cap A$, which means that $\mu(V \cap A)=\iota(\mu)(V)>0$ for all $V \in V(x)$. Thus $x \in S(\mu)$. By (IV) in $\$ 4.1, A$ is a $B^{*}$-space. $/ /$

We give a necessary and sufficient condition for a subset of a Borel measure-compact space to be Borel measure-compact.

THEOREM 4.3.3. Let $X$ be a Borel measure-compact space. Then a subset $A$ of $X$ is Borel measure-compact if and only if $\iota(\mu)$ is a regular measure on $X$ for every Borel measure $\mu$ on $A$, where $\iota$ is the natural injection from $A$ to $X$.

Proof. The 'only if' part is derived from Gardner [10, Theorem 5.4]. Conversely suppose that $\iota(\mu)$ is regular for each regular Borel measure $\mu$ on $A$; then $\iota(\mu)$ is $\tau$-smooth. For any net $\left\{F_{\alpha}\right\} \subset F(A)$ such that $F_{\alpha} \downarrow \emptyset$, we have

$$
\begin{aligned}
\inf _{\alpha} \mu\left(F_{\alpha}\right) & =\inf _{\alpha} \mu\left(\bar{F}_{\alpha} \cap A\right) \\
& =\inf _{\alpha} \iota(\mu)\left(\bar{F}_{\alpha}\right)=\iota(\mu)\left(\bigcap_{\alpha} \bar{F}_{\alpha}\right) \\
& \left.=\mu\left(\bigcap_{\alpha} \bar{F}_{\alpha}\right) \cap A\right)=\mu\left(\bigcap_{\alpha} F_{\alpha}\right)=0,
\end{aligned}
$$

which implies that $\mu$ is a $\tau$-smooth Borel measure on $A$. Hence $A$ is Borel measure-compact. //

The following lemma is easy to prove.

LEMMA 4.3.4. Let $X$ and $Y$ be topological spaces and $f$ a closed continuous map from $X$ to $Y$. If $\mu$ is a regular Borel measure on $X$, then $f(\mu)$ is a regular Borel measure on $Y$.

PROPOSITION 4.3.5. Every $F_{\sigma}$-subset of a Borel measure-compact space 
is Borel measure-compact.

Proof. Every closed subset is Borel measure-compact by Theorem 4.3.3 and Lemma 4.3.4. Then every $F_{\sigma}$-subset is Borel measure-compact by Garnder [10, Theorem 3.7]. //

Every Borel set of a Radon space is a Radon space.

For a weak Radon space, we have the following proposition by Theorem 4.2.2 and Lemma 4.3.4.

PROPOSITION 4.3.6. Every $F_{\sigma}$-subset of a weak Radon space is a weak Radon space.

As for a Borel-regular space, the following proposition is easily derived.

PROPOSITION 4.3.7. Every subset of a Borel-regular space is also a Borel-regular space.

Remark 4.3.8. (i) Example 1.2.16 shows that an open subset of a Borel measure-compact (respectively $B^{*_{-}}$, weak Radon, strongly measurecompact, measure-compact) space is not necessarily a Borel measure-compact (respectively $B^{*}$, weak Radon, strongly measure-compact, measure-compact) space.

(ii) Take $A$ in (ii) of Example 4.1.2, then $A$ is not a Radon space; that is, every subset of a Radon space is not necessarily a Radon space. 


\section{CHAPTER $V$}

\section{SUPPORTS OF BOREL MEASURES}

\subsection{Introduction}

The main purpose of this chapter is to study a new class of topological spaces where every finite Borel measure has a Lindelöf support. These spaces are called SL-spaces. We show that every metacompact space is an SL-space, furthermore a $\theta$-paracompact space is also an SL-space. However, a $\theta$-refinable space is not necessarily an SL-space. Moreover we prove that every $F_{\sigma}$-subset of an SL-space is also an SL-space, while an open subset is not always an SL-space. The class of SL-spaces is not closed by the products, though the product space of an SL-space and a $\sigma$-compact space is an SL-space.

All Borel measures in this chapter are supposed to be finite.

Now let us recall the definitions of paracompact spaces, countably paracompact spaces, metacompact spaces and $\theta$-refinable spaces.

Let $U$ and $\|$ be two covers of a set $X$; then we say that $\|$ is a refinement of $U$ if, for each $W \in W$, there exists a $U \in U$ such that $W \subset U$. If $U$ is a family of subsets of a set $X, x \in X$ and $A \subset X$, then we let

$$
\operatorname{st}(x, U)=\{U \in U: x \in U\}
$$

and

$$
\operatorname{st}(A, U)=\{U \in U: A \cap U \neq \emptyset\} \text {. }
$$

A family $u$ of subsets of a set $X$ is called point finite if $\operatorname{st}(x, U)$ is finite for each $x \in X$. A family $U$ of subsets of a topological space is called locally finite if, for every $x \in X$, there is a $V \in V(x)$ such that $s t(V, U)$ is finite. 
A topological space $X$ is paracompact (respectively metacompact) if each open cover of $X$ has a locally finite (respectively point finite) open refinement. A topological space $X$ is said to be countably paracompact if each countable open cover has a locally finite open refinement. A topological space $X$ is called $\theta$-refinable if each open cover of $X$ has an open refinement $u=U_{n \in \mathbb{N}} u_{n}$ such that $u_{n}$ covers $X$ for every $n \in \mathbb{N}$, and that given any $x \in X$ there is an $m \in \mathbb{N}$ for which $s t\left(x, u_{m}\right)$ is finite.

\subsection{Fundamental Properties of Supports}

Let $\mu$ be a Borel measure on a topological space $X$. If there exists the smallest closed subset $S^{\#}(\mu)$ which satisfies $\mu\left(S^{\#}(\mu)\right)=\mu(X)$, then we call $S^{\#}(\mu)$ the strong support of $\mu$. It is evident that $S^{\#}(\mu)=S(\mu)$ if $S^{\#}(\mu)$ exists. In general, even if a Borel measure has a non-empty support, it does not necessarily imply the existence of its strong support (see Example 1.2.15). A $\tau$-smooth Borel measure has a strong support.

When we consider all regular Borel measures, we have the following theorem by Gardner [10, Theorem 3.1). Recall that a Borel measure $\mu$ on a topological space $X$ is said to be locally measure zero if, for each $x \in X$, there exists a $V \in V(x)$ such that $\mu(V)=0$.

THEOREM 5.2.1. Let $X$ be a topological space. Then the following statements are equivalent:

(i) every non-zero regular Borel measure has a non-empty support;

(ii) every non-zero regular Borel measure has a strong support; (iii) every regular Borel measure which is locally measure zero 
is identically zero;

(iv) $X$ is Borel measure-compact.

For continuous maps and supports, we have

THEOREM 5.2.2. Let $X$ and $Y$ be topological spaces and $f$ a continuous mapping from $X$ to $Y$. If $\mu$ is a Borel measure on $X$, then $\overline{f(S(\mu))} \subset S(f(\mu))$.

Proof. We may assume that $S(\mu) \neq \varnothing$. For each $x \in S(\mu)$ and each $V \in V(f(x))$, we have

$$
f(\mu)(V)=\mu\left(f^{-1}(V)\right)>0,
$$

which implies that $f(S(\mu)) \subset S(f(\mu))$. Since $S(f(\mu))$ is closed, we have $\overline{f(S(\mu))} \subset S(f(\mu))$. /

In general, $\overline{f(S(\mu))} \neq S(f(\mu))$. In fact, under the same notation as in Example 1.2.16, we have

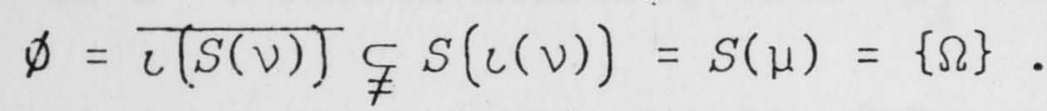

Nevertheless, the equality holds for strong supports.

THEOREM 5.2.3 (Rajput and Vakhania [39, Lemma 1]). Let $X$ and $Y$ be two topological spaces, and $\mu$ a Borel measure on $X$ with $S^{\#}(\mu) \neq \varnothing$. Then, for every continuous map $f$ from $X$ to $Y, S^{\#}(f(\mu))$ exists and $S^{\#}(f(\mu))=\overline{f\left(S^{\#}(\mu)\right)}$.

Let $A$ be a Borel measure on a subset $A$ such that $S^{\#}(\mu)$ exists; then $S^{\#}(\iota(\mu)) \cap A=S^{\#}(\mu)$ by Theorem 5.2.3, where $\iota: A \rightarrow X$ is the natural injection. In general, we have

THEOREM 5.2.4. Let $X$ be a topological space, $A$ a subset of $X$, and $\iota: A \rightarrow X$ the natural injection. If $\mu$ is a Borel measure on $A$, then $S(\iota(\mu)) \cap A=S(\mu)$.

Proof. We can assume that $S(\mu) \neq \not$. If $x \in S(\mu)$, then $\iota(\mu)(V)=\mu(V \cap A)>0$ for each $V \in V(x)$. Thus $S(\mu) \subset S(\iota(\mu))$. 
Conversely, for each $x \in S(\iota(\mu)) \cap A$ and each $V \in V(x)$, we have $\mu(V \cap A)=\iota(\mu)(V)>0$, which means that $S(\iota(\mu)) \cap A \subset S(\mu)$. //

If we consider the restrictions of Borel measures, then we have the following theorem.

THEOREM 5.2.5. Let $\mu$ be a regular Borel measure on a topological space $X$. Then the following are equivalent:

(i) $\mu$ is a $\tau$-smooth Borel measure;

(ii) for each $F \in F(X)$ with $\mu_{F}>0, S\left(\mu_{F}\right) \neq \not \supset$;

(iii) for each $F \in F(X)$ with $\mu_{F}>0, S^{\#}\left(\mu_{F}\right)$ exists;

(iv) for each subset $A$ of $X$ with $\mu_{A}>0, S\left(\mu_{A}\right) \neq \not p$;

(v) for each subset $A \subset X$ with $\mu_{A}>0, S^{\#}\left(\mu_{A}\right)$ exists.

Proof. If $\mu$ is a $\tau$-smooth measure, then $\mu_{A}$ is also $\tau$-smooth for every $A \subset X$ by Amemiya, Okada and Okazaki [2, §5]. Hence ( $i)$ implies (v), so that it suffices if we show that (ii) implies (i). Take any net $\left\{U_{\alpha}\right\} \subset O(X)$ such that $U_{\alpha} \uparrow X$. Assume that $a=\sup \mu\left(U_{\alpha}\right)<\mu(X)$. Then we can choose an increasing sequence $\left\{U_{\alpha}\right\}_{n \in \mathbb{N}}$ from $\left\{U_{\alpha}\right\}$ such that $\sup _{\alpha} \mu\left(U_{\alpha_{n}}\right)=\alpha$. Let $F=\left(U_{n \in \mathbb{N}} U_{\alpha}\right)^{c} ;$ then we have $\mu_{F}>0$, so that $S\left(\mu_{F}\right) \neq \varnothing$. Take $x \in S\left(\mu_{F}\right)$, then there is an $\alpha(x)$ such that $U_{\alpha(x)} \ni x$, which implies that $\mu\left(F \cap U_{\alpha(x)}\right)=\mu_{F}\left(F \cap U_{\alpha(x)}\right)>0$. Thus we have

$$
\begin{aligned}
a & =\mu\left(\left(\begin{array}{cc}
U & U_{\alpha} \\
n \in \mathbb{N} & \alpha_{n}
\end{array}\right) \cap U_{\alpha(x)}\right) \\
& =\mu\left(\underset{n \in \mathbb{N}}{U} U_{\alpha_{n}}\right)+\mu\left(U_{\alpha(x)} \cap F\right) \\
& >\mu\left(\underset{n \in \mathbb{N}}{U} U_{\alpha_{n}}\right)=a .
\end{aligned}
$$


This is a contradiction. Therefore $\sup _{\alpha} \mu\left(U_{\alpha}\right)=\mu(X) ;$ that is, $\mu$ is $\tau$-smooth.

Remark 5.2.6. (i) For each subset $A$ of $X, S\left(\mu_{A}\right) \subset S(\mu) \cap A$.

(ii) If $A$ is a $\mu$-thick subset or an open subset, then $S\left(\mu_{A}\right)=S(\mu) \cap A$.

(iii) Consider the restriction to supports. It follows that $S\left(\mu_{S(\mu)}\right) \subset S(\mu)$. If $S^{\#}(\mu)$ exists, then $S_{S}^{\#}(\mu)$ exists and $S^{\#}\left(\mu_{S}^{\#}(\mu)=S^{\#}(\mu)\right.$

In general, $S\left(\mu_{A}\right) \neq S(\mu) \cap A$ even if both $\mu$ and $\mu_{A}$ are Radon measures and $A$ is a closed $G_{\delta}$-subset.

EXAMPLE 5.2.7. Let $a, b, c \in \mathbb{R}$ such that $a<b<c$. Then there exists a non-negative continuous function $f$ on $\mathbb{R}$ such that

(i) $\int_{-\infty}^{\infty} f(x) d x=1$, where $d x$ is the Lebesgue measure on $\mathbb{R}$;

(ii) $[a, b]=f^{-1}(0)$.

If we let $d \mu=f d x$, then $\mu$ is a Radon measure and $\mu_{[a, c]}$ is a non-zero Radon measure. It is obvious that $a \in S(\mu)$ and $a \notin S\left(\mu_{[a, c]}\right)$; that is, we have

$$
S\left(\mu_{[a, c]}\right) \varsubsetneqq S(\mu) \cap[a, c]
$$

In general, the equality $S\left(\left.\mu\right|_{S(\mu)}\right)=S^{\prime}(\mu)$ does not hold even if $\left.\mu\right|_{S(\mu)}>0$. In fact, we have the following counter-example.

EXAMPLE 5.2.8. We use the same notation as in Example 1.2.15. Take an $a \in[0, \Omega)$. If we let $\rho=\delta_{a}+\mu$, then $S(\rho)=\{a, \Omega\}$ and $\rho_{S(\rho)}>0$. However, it follows that $\Omega \notin S\left(\rho_{S(\rho)}\right)$ and $\Omega \in S(\rho)$. Thus 
we have $S\left(\rho_{S(\rho)}\right) \varsubsetneqq S(\rho)$.

In a product space, we have

THEOREM 5.2.9. Let $\mu$ be a Borel measure on a product space $X=\prod_{\lambda \in \Lambda} x_{\lambda}$; then $S(\mu) \subset \prod_{\lambda \in \Lambda} S\left(\mu_{\lambda}\right)$, where $\mu_{\lambda}$ is the image measure $p_{\lambda}(\mu)$ by the projection $p_{\lambda}$ of $X$ to $x_{\lambda}$.

Proof. Given $x=\left(x_{\lambda}\right)_{\lambda \in \Lambda} \in S(\mu)$, we have

$$
\mu_{\lambda}\left(V_{\lambda}\right)=\mu\left(V_{\lambda} \times \prod_{\lambda^{\prime} \neq \lambda} x_{\lambda}\right)>0
$$

for each $V_{\lambda} \in V\left(x_{\lambda}\right)$, hence $x \in \prod_{\lambda \in \Lambda} S\left(\mu_{\lambda}\right)$. Thus we have $S(\mu) \subset \prod_{\lambda \in \Lambda} S\left(\mu_{\lambda}\right)$

As the following example shows, $S(\mu) \neq \prod_{\lambda \in \Lambda} S\left(\mu_{\lambda}\right)$, in general.

EXAMPLE 5.2.10. Let $d \mu(x)=(1 / 2 \pi) \exp \left(-x^{2} / 2\right) d x$ on $\mathbb{R}$. There is a non-negative continuous function $f$ on $\mathbb{R}^{2}$ vanishing outside a compact set such that $\operatorname{supp} f \varsubsetneqq p_{1}(\operatorname{supp} f) \times p_{2}(\operatorname{supp} f)$, where $p_{i}$ is the projection $(i=1,2)$ and $\operatorname{supp} f=\overline{\left\{y \in \mathbb{R}^{2}: f(y) \neq 0\right\}}$. If we let $d v=f d(\mu \otimes \mu)$, then it is easily verified that $S(\nu)=\operatorname{supp} f$ and $S\left(p_{i}(\nu)\right)=p_{i}(\operatorname{supp} f)$ $(i=1,2)$. Thus we have

$$
\begin{aligned}
S(\nu) & =\operatorname{supp} f \varsubsetneqq p_{1}(\operatorname{supp} f) \times p_{2}(\operatorname{supp} f) \\
& =S\left(p_{1}(\nu)\right) \times S\left(p_{2}(\nu)\right) .
\end{aligned}
$$

\subsection{Lindelöf Supports}

At first, we show that every Borel measure un a metacompact space has a Lindelöf support by using the idea of Moran [32]. 
THEOREM 5.3.1. Let $X$ be a metacompact space and $\mu$ a Borel measure on $X$. Then $S(\mu)$ is a Lindelöf space.

Proof. Assume that $S(\mu) \neq \varnothing$. Let $U_{0} \subset O(X)$ cover $S(\mu)$; that is, $\underset{U \in U_{0}}{U} U \supset S(\mu)$. Then there exists a point finite open refinement of $u_{0} \cup\{X \rightarrow S(\mu)\}$. Let $u_{2}=\operatorname{st}\left(S(\mu), u_{1}\right)$, and let

$$
u_{2}^{n}=\left\{U \in u_{2}: \mu(U) \geq 1 / n\right\} ;
$$

then $u_{2}=\bigcup_{n \in \mathbb{N}} u_{2}^{n}$. Suppose that the cardinal number of $u_{2}$ is uncountable. Then there is an $n \in \mathbb{N}$ such that the cardinal number of $u_{2}^{n}$ is uncountable, so that we can take an infinite sequence $\left\{P_{m}\right\}_{m \in \mathbb{N}} \subset U_{2}^{n}$. If we let $P=\overline{\operatorname{Iim}} P_{m}$, then

$$
\mu(P)=\lim _{m \rightarrow \infty} \mu\left(\bigcup_{k=m}^{\infty} P_{k}\right) \geq 1 / n,
$$

which means that $P \neq \not \supset$. Take an $x \in P$, then there is an increasing sequence $\left\{k_{s}\right\}_{s \in \mathbb{N}}$ such that $x \in \bigcap_{s=1}^{\infty} P_{k_{s}}$, which contradicts the point finiteness of $\left\{P_{k}\right\}_{k \in \mathbb{N}}$. Therefore the cardinal number of $u_{2}$ is countable. For each $U \in U_{2}$, there is a $V_{U} \in U_{0}$ such that $U \subset V_{U}$; hence we have

$$
S(\mu) \subset \underset{U \in U_{2}}{U} U \subset \underset{U \in U_{2}}{U} V_{U}
$$

Thus $S(\mu)$ is a Lindelöf space. //

COROLLARY 5.3.2. Every Borel measure on a paracompact space has a Lindelöf support.

COROLLARY 5.3.3. Suppose that a regular topological space $X$ is not paracompact but metacompact. Then there is no Borel measure of which support 
is identical to $X$.

COROLLARY 5.3.4 (Rajput and Vakhania [39, Lemma 2]). Let $\mu$ be a Borel measure on a metric space. Then $S(\mu)$ is separable.

COROLLARY 5.3.5. Let $X$ be an inseparable Banach space. Then there is no Borel measure $\mu$ such that the linear hull of $S(\mu)$ is equal to $X$. In particular, $S(\mu)$ is not equal to the unit ball.

This corollary implies Corollary 1 of Ionescu Tulcea [20].

Now we introduce a new class of topological spaces. A topological space $X$ is $\theta$-paracompact if each open cover $U$ of $X$ has an open refinement $W=\underset{n \in \mathbb{N}}{U}\left({ }_{n}\right.$ such that given $x \in X$ there is an $n(x) \in \mathbb{N}$ for which $s t\left(x, w_{n(x)}\right) \neq \varnothing$ and $s t\left(V, w_{n(x)}\right)$ is finite for some $V \in V(x)$; in other words, $w_{n(x)}$ is locally finite at $x$.

THEOREM 5.3.6. Let $\mu$ be a Borel measure on a $\theta$-paracompact space $X$. Then $S(\mu)$ is a Lindelöf space.

Proof. Assume that $S(\mu) \neq ; 0$. Take any $u \subset O(X)$ such that $u$ covers $S(\mu)$. Then there exists an open refinement $U=U_{n \in \mathbb{N}} \omega_{n}$ of $u \cup\{X-S(\mu)\}$ which satisfies the condition preceding this theorem. Let $X_{n}=\left\{x \in X: \operatorname{st}\left(x, w_{n}\right) \neq \phi, w_{n}\right.$ is locally finite at $\left.x\right\} ;$

then $X=\bigcup_{n \in \mathbb{N}} X_{n}$. Without difficulty it is shown that $X_{n}$ is open for each $n \in \mathbb{N}$. If we let

$$
u_{n}=\left\{U \cap X_{n}: U \in \operatorname{st}\left(S(\mu) \cap X_{n}, w_{n}\right)\right\},
$$

then $\mu(V)>0$ for every $V \in u_{n}$. We can show that the cardinal number of $u_{n}$ is countable for all $n \in \mathbb{N}$ by the same idea as in the proof of Theorem 5.3.4. For each $V=U \cap X_{n} \in U_{n}$, there is a $W_{V}^{n} \in U$ such that $W_{V}^{n} \supset U$. Thus we have 


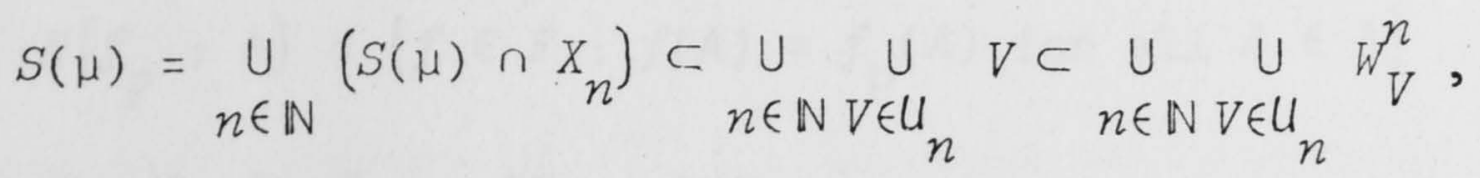

which implies that $S(\mu)$ is a Lindelöf space. //

COROLLARY 5.3.7. Let $X$ be a metacompact or $\theta$-paracompact space and $\mu$ a regular Borel measure on $X$. Then $\mu$ is $\tau$-smooth if and only if $S^{\#}(\mu)$ exists and $S^{\#}(\mu)$ is a Lindelöf space.

EXAMPLE 5.3.8. (i) The 'if' part of Corollary 5.3.7 always holds even if $X$ is neither metacompact nor $\theta$-paracompact.

(ii) The statement in Corollary 5.3.7 does not hold if we replace $S^{\#}(\mu)$ with $S(\mu)$. In fact, under the same notation as in Example 1.2.16, consider $\rho=\nu+\delta_{a}(a \in[0, \Omega))$. Then $S^{\#}(\rho)$ does not exist but $S(\rho)=\{a\}$, so that $\rho$ is a regular Borel measure on $[0, \Omega)$ of which support is compact. Nevertheless $\rho$ is not $\tau$-smooth since

$$
I=\sup _{0<\alpha<\Omega} \rho([0, \alpha))<\rho([0, \Omega))=2 .
$$

The following example was originally given by Bing [3, Example G] and Michael [27, Example 2]. Moran has treated it again in [29, IV.2] and has shown the author that a modification of the proof gives a proof that $G$ is not $\theta$-paracompact.

EXAMPLE 5.3.9. Let $P$ be an uncountable set, $Q$ the power set of $P$, and $F$ the power set of $Q$, that is, the set of all two-valued functions on $Q$. For $p \in P$ and $A \in Q$, let

$$
f_{p}(A)=\left\{\begin{array}{l}
1 \text { if } p \in A, \\
0 \text { if } p \notin A,
\end{array}\right.
$$

and let $K=\left\{f_{p}: p \in P\right\}$. We define a topology on $F$ as follows: $\left\{f_{p}\right\}$ is a neighbourhood of $f$ if $f \in F-K$; for $f_{p} \in K$ and a finite subset of $Q$, 


$$
N\left(f_{p}: \Lambda\right)=\left\{f \in F: f(A)=f_{p}(A) \text { for all } A \in \Lambda\right\}
$$

is a neighbourhood of $f_{p}$. If we let

$$
G=K u^{\prime}\{f \in F: f(A)=0 \text { for all except finitely many } A \in Q\} \text {, }
$$

then $G$ is a normal, countably paracompact, metacompact space. However, $G$ is not $\theta$-paracompact.

The following example is quoted by the referee of Okada [34].

EXAMPLE 5.3.10. Consider 'pointed extension' of $\mathbb{R}$ (see Steen and Seeback, Jr $[44,69])$. Let $X=\mathbb{R}$ as a set. We define a topology on $X$ generated by all sets $\{x\} \cup(\Phi \cap U)$, where $x \in U \in O(\mathbb{R})$. Then $X$ is a $\theta$-paracompact space, but it is not a metacompact space.

\subsection{SL-spaces}

DEFINITION 5.4.1. A topological space $X$ is called an SL-space if every Borel measure $\mu$ on $X$ has a Lindelöf support $S(\mu) \quad(S(\mu)$ may be empty).

From $\$ 5.3$, we have

THEOREM 5.4.2. A metacompact or $\theta$-paracompact space is an SL-space. Now we present a $\theta$-refinable space which is not an SL-space.

EXAMPLE 5.4.3. Let $S$ be the Sorgenfrey Zine, that is, the real line with the right half open interval topology. Then $S$ is hereditarily Lindelöf, in particular, paracompact. The product space $X=S \times S$ is separable since $\Phi \times \Phi$ is dense in $X$, but $X$ is not Lindelöf (see, for example, Steen and Seebach, Jr $[44,51,85]$ ). By Burke [6, Theorem 1.6] and Lutzer [24, Proposition 3.1] $X$ is $\theta$-refinable. There are two ways (i), (ii), to prove that $X$ is not an SL-space.

(i) Take a countable dense subset $\left\{x_{n}\right\}_{n \in \mathbb{N}}$ of $X$. If we let $\mu=\sum_{n \in \mathbb{N}} 1 / 2^{n} \delta_{x_{n}}$, then $S(\mu)=X$. Since $X$ is not a Lindelö $f$ space, $X$ 
is not an SL-space.

(ii) All Borel subsets of $X$ are Lebesgue measurable by Vitali's covering theorem (see, for example, Saks [40, Chapter 4, (3.1)]). Let $v$ be the restriction of the Lebesgue measure to $B(X)$. Since $\nu$ is still $\sigma$-finite, there exists a finite Borel measure $v_{1}$ such that $v$ is absolutely continuous with respect to $\nu_{1}$ and $\nu_{1}$ is absolutely continuous with respect to $\nu$. Hence $S\left(\nu_{1}\right)=X$, which implies that $X$ is not an SL-space.

A closed subset of an SL-space is also an SL-space. In general, we have

THEOREM 5.4.4. If $X$ is an SL-space, then every $F_{\sigma}$-subset $L$ of $X$ is also an SL-space.

Proof. We can write $L=\bigcup_{n \in \mathbb{N}} F_{n}$ for some $\left\{F_{n}\right\}_{n \in \mathbb{N}} \subset F(X)$. Let $\mu$ be a Borel measure on $L$ with $S(\mu) \neq \varnothing$. By Theorem 5.2.4, we have

$$
S(\mu)=S(\iota(\mu)) \cap L=\underset{n \in N}{U} S(\iota(\mu)) \cap F_{n},
$$

where $\iota: L \rightarrow X$ is the natural injection. Since $S(\iota(\mu)) \cap F_{n}$ is a Lindelöf space for all $n \in \mathbb{N}, S(\mu)$ is also a Lindelöf space. // An open subset of an SL-space is not always an SL-space.

EXAMPLE 5.4.5. Let $X$ be the real line with the rational sequence topology (see Steen and Seebach $[44,65]$ ). Then $X$ is a locally compact separable space, but $X$ is not a Lindelöf space. Let $Y$ denote the onepoint compactification of $X$; then $X$ is an SL-space since $X$ is compact. The open subset $X$ of $\check{X}$ is not an SL-space as (i) in Example 5.4.3.

Next we deal with products of SL-spaces. Note that the class of SL-spaces is not closed for the product. In fact, although the Sorgenfrey 
line $S$ is an SL-space, the product $S \times S$ is not an SL-space. We still have

THEOREM 5.4.6. Let $X$ be an SL-space and $Y$ a o-compact space. Then the product space $X \times Y$ is an SL-space.

Proof. Let $\mu$ be a Borel measure on $X \times Y$. By Theorem 5.2.9, we have

$$
S(\mu) \subset S\left(p_{X}(\mu)\right) \times S\left(p_{Y}(\mu)\right)
$$

where $p_{X}$ (respectively $p_{Y}$ ) is the natural projection of $X \times Y$ to $X$ (respectively $Y$ ). From the assumption, $S\left(p_{X}(\mu)\right)$ is Lindelöf and $S\left(p_{Y}(\mu)\right)$ is $\sigma$-compact. Thus $S\left(p_{X}(\mu)\right) \times S\left(p_{Y}(\mu)\right)$ is a Lindelöf space since the product of a compact space and a Lindelöf space is Lindelöf, in general. Hence $S(\mu)$ is a Lindelöf space. //

Finally we show that SL-spaces are not always transferred by continuous maps nor open maps.

EXAMPLE 5.4.7. (i) Take a topological space $Y$ which is not an SL-space. Let $X$ be the topological space which is equal to $Y$ as a set and has the discrete topology. Then the identity map from $X$ to $Y$ is a continuous bijection. Although $X$ is an SL-space, $Y$ is not an SL-Space.

(ii) Let $X=\mathbb{R}^{2}$, and let $Y=S \times S$, where $S$ is the Sorgenfrey line. Then the identity map from $X$ to $Y$ is an open bijection. Whereas $X$ is an SL-space, $Y$ is not an SL-space. 


\section{REFERENCES}

[1] W. Adamski, $\tau$-smooth Borel measures on topological spaces, Math. Nachr. 78 (1977), 97-107.

[2] I. Amamiya, S. Okada and Y. Okazaki, Pre-Radon measures on topological spaces, Kodai Math. J. 1 (1978), 101-132.

[3] R.H. Bing, Metrization of topological spaces, Canad. J. Math. 3 (1951), 175-186.

[4] S. Bochner, Harmonic Analysis and the Theory of Probability

(University of California Press, Berkeley and Los Angeles, 1955).

[5] N. Bourbaki, Integration, Chapitre 9 (Hermann, Paris, 1969).

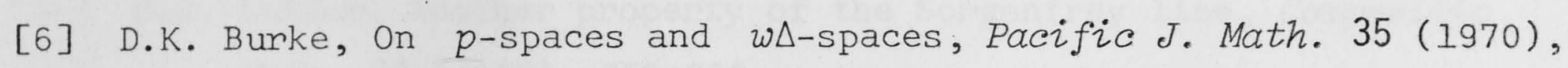
285-296.

[7] R.M. Dudley, J. Feldman and L. LeCam, On seminorms and probabilities, and abstract Wiener spaces, Ann. of Math. (2) 93 (1971), 390-408.

[8] G.A. Edgar, Measurability in a Banach space, Indiana Univ. Math. J. 26 (1977), 663-677.

[9] D.H. Fremlin, Topological Riesz Spaces and Measure Theory (University of Cambridge Press, Cambridge, 1973).

[10] R.J. Gardner, The regularity of Borel measures and Borel measurecompactness, Proc. London Math. Soc. (3) 30 (1975), 95-113.

[11] L. Gillman and M. Jerison, Rings of Continuous Functions (Van Nostrand, New York, 1960).

[12] I. Glicksberg, Stone-Čech compactifications of products, Trans. Amer. Math. Soc. 90 (1959), 369-382.

[13] G. Gruenhage and W.F. Pfeffer, When inner regularity of Borel measures implies regularity, J. London Math. Soc. (2) 17 (1978), 165-171.

[14] A.W. Hager, Approximation of real continuous functions of Lindelöf space, Proc. Amer. Math. Soc. 22 (1969), 156-163.

[15] A.W. Hager, An approximation technique for real-valued functions, General Topology and Appl. 1 (1971), 127-133.

[16] P.R. Halmos, Measure Theory (Van Nostrand, New York, 1950).

[17] D. Heidemann, on the extension of cylinder measures to $\tau$-smooth measures in linear spaces, Proc. Amer. Math. Soc. 61 (1976), $59-65$.

[18] E. Hewitt and K.A. Ross, Abstract Harmonic Analysis, Volume I (Die Grundehren der mathematischen Wissenschaften, Band 115. SpringerVerlag, Berlin, 1963). 
[19] J. Hoffmann-J $\phi$ rgensen, The Theory of Analytic Spaces (Various Publication Series No. 10. Aarhus University, Aarhus, 1970).

[20] A. Ionescu Tulcea, On pointwise convergence, compactness and equicontinuity in the lifting topology I, Z. Wahscheinlichkeitstheorie und Verw. Gebiete 26 (1973), 197-205.

[21] R.B. Kirk, Measures in topological spaces and B-compactness, Indag. Math. 31 (1969), 172-183.

[22] R.B. Kirk, Locally compact, B-compact spaces, Indag. Math. 31 (1969), 333-344.

[23] J.D. Knowles, Measures on topological spaces, Proc. London Math. Soc. (3) 17 (1967), 139-156.

[24] D.J. Lutzer, Another property of the Sorgenfrey line, Compositio Math. 24 (1972), 359-363.

[25] J. Marík, The Baire and Borel measures, Czechoslovak Math. J. 7 (82) (1957), 248-253.

[26] P. Massani, Measurability and Pettis integration in Hilbert spaces, Proceedings of the Measure Theory Conference (Lecture Notes in Mathematics, 541, 69-105. Springer-Verlag, Berlin, 1976).

[27] E. Michael, Point finite and locally finite coverings, Canad. J. Math. 7 (1955), 275-279.

[28] I. Mitoma, S. Okada and Y. Okazaki, Cylindrical o-algebra and cylindrical measure, Osaka J. Math. 14 (1977), 635-647.

[29] W. Moran, Measures on completely regular spaces ( $\mathrm{PhD}$ thesis, University of Sheffield, Sheffield, 1968).

[30] W. Moran, The additivity of measures on completely regular spaces, $J$. London Math. Soc. 43 (1968), 633-639.

[31] W. Moran, Measures and mappings on topological spaces, Proc. London Math. Soc. (3) 19 (.1969), 493-503.

[32] W. Moran, Measures on metacompact spaces, Proc. London Math. Soc. (3) 20 (1970), 507-524.

[33] S.E. Mosiman and R.F. Wheeler, The strict topology in a completely regular setting: Relations to topological measure theory, Canad. J. Math. 24 (1972), 873-890.

[34] S. Okada, Supports of Borel measures, J. Austral. Math. Soc. Ser. A (to appear).

[35] S. Okada and Y. Okazaki, On measure-compactness and Borel measurecompactness, Osaka J. Math. 15 (1978), 183-191. 
[36] S. Okada and Y. Okazaki, Projective limit of infinite Radon measures, J. Austral. Math. Soc. Ser. A 25 (1978), 328-331.

[37] D.B. Pollard, Topological measure theory with applications to probability ( $\mathrm{PhD}$ thesis, Australian National University, Canberra, 1976).

[38] Yu.V. Prokhorov, The method of characteristic functionals, Proceedings of the Fourth Berkeley Symposium of Mathematics, Statistics and Probability (University of California Press, Berkeley: and Los Angeles, 1961, 403-419).

[39] B.S. Rajput and N.N. Vakhania, On the support of Gaussian probability measures on locally convex topological vector spaces, Multivariate Analysis - IV (Proc. Fourth Intern. Sympos. Multivariate Analysis, 1975, 297-309. North-Holland, Amsterdam, 1977).

[40] S. Saks, Theory of the Integral (Hafner, Warsaw, 1937).

[41] W. Schachermayer, Measures cylindriques sur les espaces de Banach qui ont le propriété de Radon-Nikodym, C.R. Acad. Sci. Paris, Sér. A 282 (1976), 227-229.

[42] W. Schachermayer, Zylindrische Masze und die Radon-Nikodym-Eigenschaft von Banach-Räumen (PhD thesis, Universität Wien, Wien, 1976).

[43] L. Schwartz, Radon Measures on Arbitrary Topological Spaces and Clindrical Measures (University of Oxford Press, London, 1973).

[44] L.A. Steen and J.A. Seebach, Jr, Counterexamples in Topology (Holt, Rinehart and Winston, New York, 1970).

[45] A. Tortrat, Sur les lois t-régulières, leur produits et leur convolution, Transactions of the Sixth Prague Conference on Information Theory, Statistical Decision Functions, Random Processes, Prague, 1971 (Academia, Publishing House of the Czechoslovak Academy of Sciences, Prague, 1973).

[46] A. Tortrat, Prolongements t-réguliers, Applications aux probabilités gaussiennes, preprint.

[47] V.S. Varadarajan, Measures on topological spaces, Amer. Math. Soc. Trans2. (2) 78 (1965), 161-228.

[48] J.M. Worrel, In and H.H. Wicke, Characterization of developable topological spaces, Canad. J. Math. 17 (1965), 820-830.

[49] Y. Yamasaki, Kolmogorov's extension theorem for infinite measures, Publ. RIMS, Kyoto Univ. 10 (1975), 381-411. 